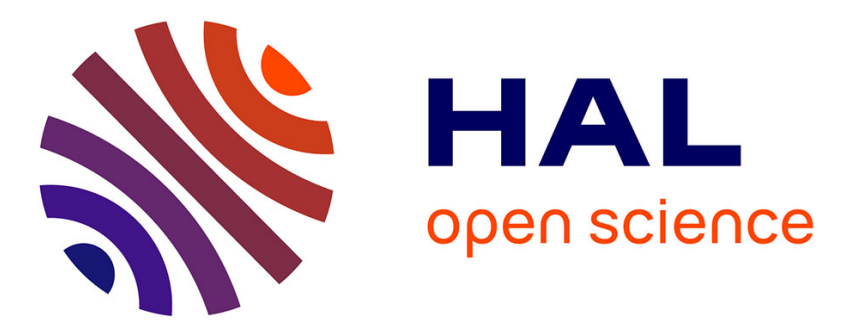

\title{
Cross-comparison of global simulation models applied to Mercury's dayside magnetosphere
}

Sae Aizawa, Léa S Griton, Shahab Fatemi, Willi Exner, Jan Deca, Filippo Pantellini, Manabu Yagi, Daniel Heyner, Vincent Génot, Nicolas André, et al.

\section{- To cite this version:}

Sae Aizawa, Léa S Griton, Shahab Fatemi, Willi Exner, Jan Deca, et al.. Cross-comparison of global simulation models applied to Mercury's dayside magnetosphere. Planetary and Space Science, 2021, 198 (April), pp.105176. 10.1016/j.pss.2021.105176 . hal-02551111v3

HAL Id: hal-02551111

https://hal.science/hal-02551111v3

Submitted on 2 Feb 2021

HAL is a multi-disciplinary open access archive for the deposit and dissemination of scientific research documents, whether they are published or not. The documents may come from teaching and research institutions in France or abroad, or from public or private research centers.
L'archive ouverte pluridisciplinaire HAL, est destinée au dépôt et à la diffusion de documents scientifiques de niveau recherche, publiés ou non, émanant des établissements d'enseignement et de recherche français ou étrangers, des laboratoires publics ou privés.

\section{(ㄷ)(1) $\$$}

Distributed under a Creative Commons Attribution - NonCommerciall 4.0 International 


\section{Journal Pre-proof}

Cross-comparison of global simulation models applied to Mercury's dayside magnetosphere

S. Aizawa, L.S. Griton, S. Fatemi, W. Exner, J. Deca, F. Pantellini, M. Yagi, D. Heyner, V. Génot, N. André, J. Amaya, G. Murakami, L. Beigbeder, M. Gangloff, M.

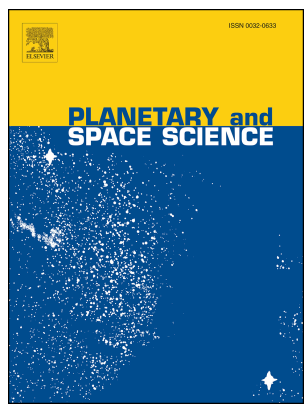
Bouchemit, E. Budnik, H. Usui

PII: S0032-0633(21)00015-5

DOI: https://doi.org/10.1016/j.pss.2021.105176

Reference: PSS 105176

To appear in: Planetary and Space Science

Received Date: 5 June 2020

Revised Date: 23 November 2020

Accepted Date: 27 January 2021

Please cite this article as: Aizawa, S., Griton, L.S., Fatemi, S., Exner, W., Deca, J., Pantellini, F., Yagi, M., Heyner, D., Génot, V., André, N., Amaya, J., Murakami, G., Beigbeder, L., Gangloff, M., Bouchemit, M., Budnik, E., Usui, H., Cross-comparison of global simulation models applied to Mercury's dayside magnetosphere, Planetary and Space Science, https://doi.org/10.1016/j.pss.2021.105176.

This is a PDF file of an article that has undergone enhancements after acceptance, such as the addition of a cover page and metadata, and formatting for readability, but it is not yet the definitive version of record. This version will undergo additional copyediting, typesetting and review before it is published in its final form, but we are providing this version to give early visibility of the article. Please note that, during the production process, errors may be discovered which could affect the content, and all legal disclaimers that apply to the journal pertain.

(c) 2021 Published by Elsevier Ltd. 


\section{Cross-comparison of global simulation models applied to}

3 Mercury's dayside magnetosphere

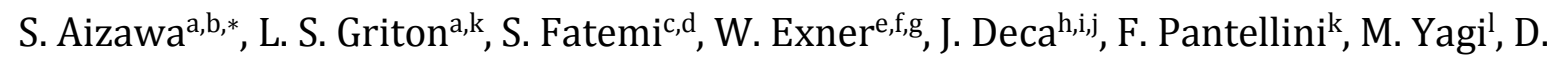
Heynere $^{\mathrm{e}}$, V. Génot ${ }^{\mathrm{a}}$, N. Andréa, J. Amayam ${ }^{\mathrm{m}}$, G. Murakamin ${ }^{\mathrm{n}}$, L. Beigbeder ${ }^{\mathrm{o}}$, M. Gangloffa, M. Bouchemit $^{\mathrm{a}}$, E. Budnik ${ }^{\mathrm{p}}$, H. Usuiq

${ }^{a}$ IRAP, CNRS-CNES-UPS, Toulouse, France

${ }^{\mathrm{b}}$ Graduate School of Science, Tohoku University, Sendai, Japan

${ }^{\mathrm{c}}$ Swedish Institute of Space Physics, Kiruna, Sweden

${ }^{\mathrm{d}}$ Department of Physics at Ume a University, Ume a, Sweden

e Institute for Geophysics and extraterrestrial Physics, Technische Universit"at Braunschweig, Braunschweig, Germany

fInstitute for Theoretical Physics, Technische Universiẗat Braunschweig, Braunschweig, Germany

${ }^{g}$ School of Earth and Atmospheric Sciences, Georgia Institute of Technology, Atlanta, USA

${ }^{h}$ Laboratory for Atmospheric and Space Physics (LASP), University of Colorado Boulder, Boulder, Colorado 80303, USA

institute for Modeling Plasma, Atmospheres and Cosmic Dust, NASA/SSERVI, California 94035, USA

jLaboratoire Atmosph`eres, Milieux, Observations Spatiales (LATMOS), Universit'e de Versailles `a Saint Quentin, 78280 Guyancourt, France

${ }^{\mathrm{k}}$ LESIA, Observatoire de Paris, Universit'e PSL, CNRS, Sorbonne Universit'e, Universit'e de Paris, 5 place Jules Janssen, 92195 Meudon, France

${ }^{1}$ RIKEN, Kobe, Japan

${ }^{\mathrm{m}} \mathrm{CmPA}$, Mathematics Department, KU Leuven, Belgium

${ }^{\mathrm{n}}$ ISAS/JAXA, Sagamihara, Japan

${ }^{\circ} \mathrm{GFI}$, Toulouse, France

${ }^{\mathrm{p}}$ Noveltis, Toulouse, France

${ }^{\mathrm{q}}$ Kobe University, Kobe, Japan

Sae AIZAWA: Conceptualization, Visualization, writing-original draft preparation, writing - review \& editing

Lea S. Griton : Conceptualization, Methodology, validation, writing-original draft preparation, writing - review \& editing

S. Fatemi: Conceptualization, Methodology, validation, writing-original draft preparation, writing - review \& editing

W. Exner : Conceptualization, Methodology, validation, writing-original draft preparation, writing - review \& editing

37 J. Deca : writing-original draft preparation, writing - review \& editing

38 F. Pantellini : Methodology, validation, writing-original draft preparation, writing review \& editing

M. Yagi: Methodology, writing-original draft preparation, writing - review \& editing 
42 D. Heyner: Supervision, writing - review \& editing

43 V. Génot: writing - review \& editing, Methodology

$44 \quad$ N. André: writing - review \& editing, funding acquisition

45 J. Amaya : writing-original draft preparation

46 G. Murakami : Supervision

47 H. Usui: Supervision

48 L. Beigbeder, M. Gangloff, M. Bouchemit, E. Budnik : Technical support on 49 Methodology 


\title{
Cross-comparison of global simulation models applied to Mercury's dayside magnetosphere
}

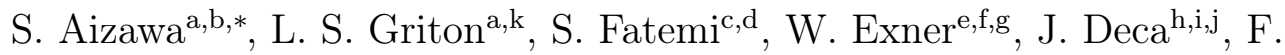 \\ Pantellini $^{\mathrm{k}}$, M. Yagi ${ }^{1}$, D. Heyner ${ }^{\mathrm{e}}$, V. Génot ${ }^{\mathrm{a}}$, N. Andréa ${ }^{\mathrm{a}}$, J. Amaya ${ }^{\mathrm{m}}$, G. \\ Murakami $^{\mathrm{n}}$, L. Beigbeder ${ }^{\mathrm{o}}$, M. Gangloff ${ }^{\mathrm{a}}$, M. Bouchemit ${ }^{\mathrm{a}}$, E. Budnik ${ }^{\mathrm{p}}, \mathrm{H}$. \\ Usui $^{\mathrm{q}}$ \\ ${ }^{a} I R A P, C N R S-C N E S-U P S$, Toulouse, France \\ ${ }^{b}$ Graduate School of Science, Tohoku University, Sendai, Japan \\ ${ }^{c}$ Swedish Institute of Space Physics, Kiruna, Sweden \\ ${ }^{d}$ Department of Physics at Umeå University, Umeå, Sweden \\ ${ }^{e}$ Institute for Geophysics and extraterrestrial Physics, Technische Universität \\ Braunschweig, Braunschweig, Germany \\ ${ }^{f}$ Institute for Theoretical Physics, Technische Universität Braunschweig, Braunschweig, \\ Germany \\ ${ }^{g}$ School of Earth and Atmospheric Sciences, Georgia Institute of Technology, Atlanta, \\ USA \\ ${ }^{h}$ Laboratory for Atmospheric and Space Physics (LASP), University of Colorado \\ Boulder, Boulder, Colorado 80303, USA \\ ${ }^{i}$ Institute for Modeling Plasma, Atmospheres and Cosmic Dust, NASA/SSERVI, \\ California 94035, USA \\ ${ }^{j}$ Laboratoire Atmosphères, Milieux, Observations Spatiales (LATMOS), Université de \\ Versailles à Saint Quentin, 78280 Guyancourt, France \\ ${ }^{k}$ LESIA, Observatoire de Paris, Université PSL, CNRS, Sorbonne Université, Université \\ de Paris, 5 place Jules Janssen, 92195 Meudon, France \\ ${ }^{l}$ RIKEN, Kobe, Japan \\ ${ }^{m}$ CmPA, Mathematics Department, KU Leuven, Belgium \\ ${ }^{n} I S A S / J A X A$, Sagamihara, Japan \\ ${ }^{\circ}$ GFI, Toulouse, France \\ ${ }^{p}$ Noveltis, Toulouse, France \\ ${ }^{q}$ Kobe University, Kobe, Japan
}

\section{Abstract}

We present the first comparison of multiple global simulations of the so-

\footnotetext{
${ }^{*}$ Corresponding author.

Email address: sae.aizawa@irap.omp.eu (S. Aizawa)
} 
lar wind interaction with Mercury's dayside magnetosphere, conducted in the framework of the international collaborative project SHOTS - Studies on Hermean magnetosphere Oriented Theories and Simulations. Two 3D magnetohydrodynamic and two 3D hybrid simulation codes are used to investigate the global response of the Hermean magnetosphere without its exosphere to a northward-oriented interplanetary magnetic field. We cross-compare the results of the four codes for a theoretical case and a MESSENGER orbit with similar upstream plasma conditions. The models agree on bowshock and magnetopause locations at $2.1 \pm 0.1$ and $1.4 \pm 0.08$ Mercury planetary radii, respectively. The latter locations may be influenced by subtle differences in the treatment of the plasma boundary at the planetary surface. The predicted magnetosheath thickness varies less between the codes. Finally, we also sample the plasma data along virtual trajectories of BepiColombo's Magnetospheric and Planetary Orbiter. Our ability to accurately predict the structure of the Hermean magnetosphere aids the analysis of the onboard plasma measurements of past and future magnetospheric missions.

Keywords: Mercury, BepiColombo, Modeling

\section{Introduction}

$2 \quad$ Mercury is a planet of extremes that is continuously battered by a harsh and dynamic solar wind. Combined with interplanetary magnetic field (IMF) 4 magnitudes up to $30 \mathrm{nT}$ and a small Parker spiral angle, Mercury's weak internal magnetic field produces a unique magnetosphere in the solar sys6 tem $[1,2,3,4,5]$. For example, the Hermean magnetosphere is twenty times 7 smaller in volume than Earth's magnetosphere and Mercury's volume frac- 
tion in its magnetosphere is approximately five hundred times larger than the equivalent terrestrial number Slavin et al. [6], Slavin et al. [7, 8]. As a result, the solar wind can directly interact with the surface, even outside the magnetospheric cusps [9]. In addition, the significant offset between the magnetic dipole origin and the center of the planet results in a surface magnetic field strength in the northern hemisphere that is double the nominal value estimated for the southern hemisphere [10, 11, 12, 13]. These particularities culminate in fascinating particle precipitation patterns and differential space weathering that is as variable as the upstream solar wind $[14,15,16]$. The absence of a significant ionosphere makes Mercury's conductive core an integral part of the electrodynamic current closure and complicates the evolution of the complex local plasma environment even more $[17,18]$.

Numerical simulations of the solar wind interaction with the Hermean magnetosphere have thus far adopted multi-fluid/magnetohydrodynamic [19, 20, 17, 21] and hybrid approaches (representing the ions as computational particles and the electron populations as a (massless) fluid) [22, 23, 24, 25, 26, 27, 28, 29, 30, 31, 32, 16, 33]. These models, designed to focus on the ion kinetics, have been successful in recreating the general structure of Mercury's local plasma environment. For example, with a 3D hybrid model, Müller et al. [34] characterised a diamagnetic current system that originates from the proton pressure gradients at Mercury's inner magnetosphere to explain the day- and night-side diamagnetic decreases observed by MESSENGER [6, 35]. In addition, recent numerical developments have produced the first fully kinetic, global simulations of the Hermean magnetosphere [36, 37, 38]. 
Due to mission constraints such as limited field of view and energy range to detect ions, both the Mariner-10 and MESSENGER spacecraft were limited to measure the Hermean plasma environment and thus, to fully disentangle plasma processes such as finite-gyroradius effects and complex electron dynamics [4]. Complementary to the previous missions, BepiColombo's Magnetospheric (Mio) [39]) and Planetary Orbiter (MPO) [40]) allow for multi-spacecraft coordinated observations [41]. Their plasma instruments focus on direct measurements of the response of Mercury's magnetosphere and its near-space environment to dynamic changes in the solar wind, including plasma-wave-charged-particle resonances, kinetic-scale instabilities and particle distributions, and energy transfer via field-aligned currents and waves [4].

In order to optimally prepare for the measurement campaign and to be able to fully interpret and analyse the data during the forthcoming Mercury flybys and during the orbital phase, or in other words, to exploit most efficiently the multi-point measurements allowed by the dual spacecraft and the synergies between the various sensors of the onboard plasma suite, sophisticated modelling tools are required. Hence, the SHOTS (Studies on Hermean magnetosphere Oriented Theories and Simulations) project has been established as an integral part of the BepiColombo Young Scientist Working Group. Its aim is to share and compare simulations results among the BepiColombo Science Working Team in order to prepare the scientific analysis of the in-situ magnetospheric observations gathered by Mio and MPO. 
In this first comparative study, we identify the differences between fluid and hybrid simulation approaches to model the structure of the Hermean magnetosphere and its plasma environment. We compare the bow shock and magnetopause locations with a representative set of MESSENGER measurements and predict the plasma environment along virtual trajectories of BepiColombo's Mio and MPO spacecraft.

\section{Model descriptions and methodology constraints}

The four computer models used in this comparison study are briefly described here, with special emphasis on their inner boundary conditions. We find that subtle differences in the treatment of the plasma boundary at the planetary surface affect the global solar wind - magnetosphere structure. Two magnetohydrodynamic (MHD) and two hybrid codes have been employed to identify the consequences of the different assumptions that are implemented in the physical model of the codes. In Table 1, we summarize the general numerical settings adopted for the four codes, such as the number of cells used, the size of the simulation domain, the spatial resolution, and the time step. The output from all models are stored and discussed using the Mercury Solar Orbital (MSO) frame. The X-axis points towards the Sun and the Y-axis is chosen opposite to the orbital motion of Mercury. The Z-axis points to the geophysical north and completes the right-handed coordinate system. The intrinsic magnetic field of the planet is set as a dipole with a $480 \mathrm{~km}$ offset towards the north from the planetary center [42]. The dipole 
moment is $200 \mathrm{nT} \times \mathrm{R}_{\mathrm{M}}^{3}$. To avoid further numerical complexities, we do not include the tenuous Mercury's exosphere in our simulations just yet. In a recent hybrid study, Exner et al. [33] investigated the kinetic effects of different sodium exosphere surface densities onto the Hermean magnetsphere. Authors found that an average sodium exosphere based on a realistic model of [cite Gamborino Monte-Carlo Paper] does not pose a significant factor to the magnetic field structure inside the magnetosphere. Therefore, we do not include the tenuous sodium exosphere in this study.

\subsection{MHD models}

The three-dimensional MPI-AMRVAC (MPI-Adaptive Mesh Refinement Versatile Advection Code) code (hereafter AMRVAC) is the first three-dimensional MHD model we use in this study. This code integrates the MHD equations using a two-step Lax-Friedrichs-type scheme associated with a Woodward gradient limiter $[43,44]$. A Powell correction is also used to satisfy the $\nabla \cdot \mathbf{B}=0$ condition at each time step [45]. In order to limit magnetic diffusion, the magnetic field $\mathbf{B}$ is split into an analytically prescribed background field $\mathbf{B}_{\mathbf{0}}$ and a residual field $\mathbf{B}_{1}$ [46]. The full system of equations is solved on a spherical grid that is linearly spaced along the angular coordinates $\theta$ and $\phi$ and logarithmically spaced along the radial coordinate $r$. Hence, the simulation domain itself is a spherical shell. Note from Table 1 that only AMRVAC employs a spherical coordinate system. With a total number of cells of $\left(N_{r}, N_{\theta}, N_{\phi}\right)=(36,36,72)$, covering the radial interval $r \in R_{\mathrm{M}}[1,10]$, the horizontal and vertical cell size near the surface are $213 \mathrm{~km}$ and $161 \mathrm{~km}$, respectively. At the outer boundary, free slip conditions $(\partial / \partial r=0)$ are ap- 
plied to all fields where the angle between the solar wind direction and the normal to the boundary direction is $<80^{\circ}$. Ambient (upstream) solar wind conditions are set at the remaining sides of the domain. At the inner boundary of the simulation domain, here the planetary surface, the radial velocity is set to zero. A free slip condition is applied to the tangential components of the momentum $\rho \mathbf{v}$. If the radial velocity immediately above the surface is positive (i.e. in case of outflow), the plasma number density and the total fluid pressure are set to $n=30 \mathrm{~cm}^{-3}$ and $p=0.1 \mathrm{nPa}$, respectively. On the other hand, if the radial velocity immediately above the surface is negative (i.e. in case of inflow), $n$ and $p$ are allowed to evolve within $15-150 \mathrm{~cm}^{-3}$ and $0.1-1 \mathrm{nPa}$. A free slip condition is also applied to the normal component of $\mathbf{B}_{1}$ at the surface. The tangential components are set to zero. The results of AMRVAC are interpolated to a uniform Cartesian grid with a spatial resolution of $81.5^{3} \mathrm{~km}^{3}$ for ease of comparison with the other simulation models.

Yagi's code is the second three-dimensional MHD model we use [47, 48]. In this code, a Rational-CIP (Constrained Interpolation Profile) algorithm is implemented to solve the advection term [48] and a fourth-order RungeKutta and a fourth-order central difference method are used to solve the non-advection terms needed to advance the numerical scheme in time and space. In contrast to AMRVAC, the magnetic vector potential $\mathbf{A}$ is computed instead of the magnetic field, ensuring $\nabla \cdot \mathbf{B}=0$ by definition and a uniform Cartesian grid is adopted. In this study, the grid resolution is set to $122 \mathrm{~km}^{3}$. The simulation domain measures $(-10:+6, \pm 5, \pm 5) \mathrm{R}_{\mathrm{M}}$, with the number of cells of $(L x, L y, L z)=(300,300,300)$. The inner boundary of 
the computational domain is the planetary surface. Mercury is treated as a reflective obstacle, allowing no flux to penetrate across the surface (obstacle). We also apply the free slip condition which does not allow any radial pressure and density gradient at the planetary surface. The inner boundary conditions accommodate a smooth convection of the magnetic field in the vicinity of the planet, mimicking obstacle with low conductivity.

\subsection{Hybrid models}

AIKEF (Adaptive Ion Kinetic Electron Fluid) is the first three-dimensional hybrid model we employ in this study. Contrary to MHD, a hybrid model treats ions kinetically while electrons are included as a massless chargeneutralizing fluid. In this study, the number of computational macro-particle per cell is set to 25 to represent their Maxwellian velocity distribution. The magnetic and electric fields are obtained by solving the Maxwell equations, i.e., the electric field $\mathbf{E}$ is directly calculated from the electron momentum equation and Faraday's law, $\partial \mathbf{B} / \partial t=-\nabla \times \mathbf{E}$, is used to advance the magnetic field $\mathbf{B}$ in time. The fields propagate between the grid points with a Runge-Kutta-algorithm. AIKEF operates on a Cartesian grid and the mesh is capable of automatically adapting its resolution in regions where large field gradients exist $[49,32]$. For simplicity, we do not activate this function here and use a fixed spatial resolution of $100 \mathrm{~km}^{3}$ instead, and the simulation domain is set to $(-6:+8, \pm 9, \pm 9) \mathrm{R}_{\mathrm{M}}$ with the number of cells of $(L x, L y, L z)=(320,224,224)$. To include magnetic induction processes within Mercury, Mercury's core is assumed to be highly conductive leading the resistivity in the core region is set to zero. The magnetic diffusion in the 
mantle is facilitated by applying a maximum resistivity of $1.21 \cdot 10^{7} \Omega \cdot m$ $[17,32]$ and employing a Crank-Nicolson algorithm. The resistivity profile is smoothed at the surface and core-mantle-boundary, identical to Jia et al. [17]. Mercury's surface is treated as a perfect plasma absorber, i.e., particles impacting the surface are removed. To ensure numerical stability, $0.2 \%$ of smoothing parameter is applied between the grid points [50].

The Amitis is a GPU-based (Graphics Processing Units) three-dimensional hybrid model of plasma that currently runs only on a single CPU-GPU pair [30]. It has been developed to reduce the computational resources that are typically needed for running global simulations and resulted in performance enhancement of 10x-100x over its CPU-based predecessor. Identical to AIKEF, the model kinetically tracks positively charged macro-particles, i.e., the ion population, by solving the Lorentz equation of motion while using a fluid description for mass-less electrons. The electric field and magnetic field are obtained by solving Maxwell equations. The model is grid-based and uses regular-spaced, cell-center Cartesian grids to solve all the equations. We choose a spatial resolution of $125 \mathrm{~km}^{3}$ with 16 macro-particles per cell, and simulation domain is set to $( \pm 7, \pm 10, \pm 10) \mathrm{R}_{\mathrm{M}}$. The model self-consistently couples the geophysical, induced electromagnetic response of the interior of a planetary body to the electromagnetic response of the incident plasma and magnetic fields by solving Maxwell's equations for the plasma and a magnetic diffusion equation $\left(\partial \mathbf{B} / \partial t=-\nabla \times \nabla \times \mathbf{B} / \mu_{0} \sigma\right)$ for the interior of the object, where $\mu_{0}$ is the permeability of free space and $\sigma$ is the conductive profile for the interior of the planetary body [30]. Amitis adopts periodic 
outer boundary conditions for its particles and electromagnetic fields along the axes perpendicular to the solar wind flow. Parallel to the flow the code continuously injects solar wind ions at the most upstream grid cell. The downstream boundary, identical to its implementation of the planetary surface, is a perfect plasma absorber. Unlike AIKEF, a smoothing routine is not applied in Amitis.

When plasma impacts onto the surface, a vacuum is formed in the downstream regions. In contrast to MHD codes where a minimum charge density is present in these regions of vacuums to enable the continued calculation of the field equations, hybrid models need special handlings of the vaccum regions. AIKEF employs ghost-ions with the same charge-to-mass ratio as solar wind ions but in tenuous densities to propagate the electric and magnetic fields in the vacuum regions. This approach enables a smooth density profile at the vacuum region edges without large gradients, while also increasing the numerical resource usage due to the handling of an additional species Trávníček et al. [25]. A different approach is used in Amitis, where, each time step, the cells within the vacuum regions obtain a flat resistivity value. This approach allows for the emergence of localized currents that propagate the electric and magnetic fields instead of ions. The vacuum state of the cells is checked every time step which does not increase numerical resources by a significant amount.However, this approach leads to unphysical resistivity gradients throughout the downstream regions that might lead to unphysical currents. Therefore, the resistivity value is capped at $10^{7} \Omega \mathrm{m}$ so limit the resistivity gradients. As both approaches result in good agreement with 
Table 1: Summary of the numerical settings. For AMRVAC the finest spatial resolution is quoted.

\begin{tabular}{ccccccc}
\hline & Grid type & $\#$ cells & Domain size & Resolution & Time step & particles/cell \\
\hline AMRVAC [43] & Spherical & $(36,36,72)$ & $r \in R_{\mathrm{M}}[1,10]$ & $213 \times 161 \mathrm{~km}$ & $0.03 \mathrm{sec}$ & - \\
Yagi [47] & Cartesian & $(300,300,300)$ & $(-10:+6, \pm 5, \pm 5) R_{\mathrm{M}}$ & $122 \mathrm{~km}$ & $0.02 \mathrm{sec}$ & - \\
AIKEF [49] & Cartesian & $(320,224,224)$ & $(-6:+8, \pm 9, \pm 9) R_{\mathrm{M}}$ & $100 \mathrm{~km}$ & $0.08 \mathrm{sec}$ & 25 \\
Amitis [30] & Cartesian & $(234,396,396)$ & $( \pm 7, \pm 10, \pm 10) R_{\mathrm{M}}$ & $125 \mathrm{~km}$ & $0.001 \mathrm{sec}$ & 16 \\
\hline
\end{tabular}

spacecraft data $[32,31]$, we do not investigate how these approaches relate to each other in this study.

\subsection{Common visualization tools}

We use netCDF as a multidimensional format with meta information for all simulations. It allows us to have a unified format for comparative visualizations, data interoperability and reusability. In the present study, we employ Paraview for 3D data visualization and analysis. In addition, we use 3Dview and AMDA (Automated Multi-Dataset Analysis), which makes use of the SPASE (Physics Archive Search and Extract) simulation data model $[51,52,53,54]$. Using SPICE kernels, 3Dview is a 3D JAVA tool that provides visualizations of the positions and attitudes of planetary missions and bodies in combination with observational data, simulations, and analytical models. AMDA is an online database and analysis tool in which in-situ observations, ground based observations, and models can be browsed, manipulated and downloaded (a workspace is available for each user). AMDA and 3Dview are developed by the CDPP (Centre de Données de la Physique des Plasmas) and available to contributing developers under a GPLv3 licence. 


\section{Science cases}

In this work we discuss two cases: (a) a classical textbook case under purely northward IMF conditions to obtain the characteristics of each code, and (b) a direct comparison with a pre-selected MESSENGER orbit.

\subsection{Case a: Northward IMF}

Northward IMF conditions are chosen to anticipate a stable dayside magnetosphere structure. Typically, a southward IMF gives rise to more unstable magnetosphere conditions as continuous dayside magnetic reconnection in combination with a relatively short Dungey cycle does not allow the system to relax [12]. In the case of a northward IMF, the reconnection sites move to high latitudes near the magnetospheric cusps.

We set the typical solar wind condition around 0.3 AU, e.g., IMF magnitude to $20 \mathrm{nT}$ and adopt a solar wind proton density of $30 \mathrm{~cm}^{-3}$, an Alfvén Mach number of 5 and a total plasma beta $(\beta)$ of 1.3 . The solar wind speed measures $400 \mathrm{~km} \mathrm{~s}^{-1}[55,9]$. The MHD models assume a total density equal to the proton density, whereas the plasma temperature $(43 \mathrm{eV})$ is set to the sum of the electron $(21.5 \mathrm{eV})$ and proton $(21.5 \mathrm{eV})$ temperatures (Table 2) [56, 57].

\subsection{Case b: MESSENGER comparison}

We select a MESSENGER orbit that allows a simulation setup as close as possible to our theoretical northward IMF case. Due to its mid-day to midnight orientation (X-Z plane), orbit 1415 (November 8, 2012) provides a 
Table 2: Summary of the common input physical and plasma parameters. $S W$ denote the solar wind.

\begin{tabular}{lcc} 
& Case (a) Northward IMF & Case (b) MESSENGER \\
\hline Planetary radius $R_{M}[\mathrm{~km}]$ & \multicolumn{2}{c}{2440} \\
Planetary dipole moment $\left[\mathrm{nT} \times R_{M}^{3}\right]$ & 200 \\
Northward dipole offset $[\mathrm{km}]$ & & 480 \\
\hline SW proton density $\left[\mathrm{cm}^{-3}\right]$ & 30 & 40 \\
SW proton + electron temperature $[\mathrm{eV}]$ & $21.5+21.5$ & $12+18$ \\
SW total plasma $\beta$ & 1.3 & 0.69 \\
SW Alfvén Mach number & 5 & 5 \\
SW plasma velocity $\left[\mathrm{km} \mathrm{s}^{-1}\right]$ & 400 & 459 \\
SW Magnetosonic Mach number & 3.5 & 8.5 \\
IMF $(X, Y, Z)$ components $[\mathrm{nT}]$ & $(0,0,+20)$ & $26,-6,4.9)$ \\
IMF magnitude $[\mathrm{nT}]$ & 20 & 26.6 \\
\hline
\end{tabular}

close comparison. The orbit has a Disturbance Index of less than 25 [58], the lowest magnetic activity quartile, the Z-components of the IMF at the inbound an outbound bow shock crossings are positive and within $2 \mathrm{nT}$ of each other, and the IMF variability along the entire orbit is less than $10 \mathrm{nT}$. Combining these parameter values indicate stable solar wind conditions.

We use the inbound part of the orbit to compute the average IMF vector to be inserted in our models. Further, the Alfvén Mach number is set to 5, the solar wind speed equals $459 \mathrm{~km} \mathrm{~s}^{-1}$ and the proton and electron temperature measure $12 \mathrm{eV}$, and $18 \mathrm{eV}$, respectively [56, 59]. 


\section{Results}

\subsection{Case a: Northward IMF}

A purely northward IMF configuration provides a stable magnetosphere configuration. We let each model run for 5 minutes, that is, 5 Dungey cycles, to reach their respective quasi-stationary states.. Figure 1 shows the solar wind proton density maps in the $X-Z$ (top) and $X-Y$ (bottom) plane on a logarithmic scale, including also velocity lines. The two left panels are the results from the MHD codes while the two right panels present the results from the hybrid codes. All models have converged to a very similar global structure for the Hermean magnetosphere and the characteristic features are present: the shape of bowshock, the higher density magnetosheath, and the magnetospheric cusps. However, the models do not agree on the proton density near the planet and inside the nightside magnetosphere. We also observe different locations of the magnetopause structure.

The $X-Z$ cut of AMRVAC (Figure 1, upper left-most panel) shows a less sharp shock structure at the bow shock, a low density dayside magnetosphere, patches of higher density close to the surface in the cusp (which is defined by the magnetic field topology) and tail regions, and an asymmetric structure for the nightside magnetosphere. The latter is uniquely present in AMRVAC. The model also has the largest magnetopause flaring angle among the four models. The $X-Y$ cut of AMRVAC (bottom left-most panel) shows the symmetric structure in the dawn-dusk plane. Yagi's MHD code in the $X-Z$ plane (upper middle-left panel) presents a sharper bow shock as compared to AMRVAC, most likely due to the model's less diffusive scheme. In addition, 

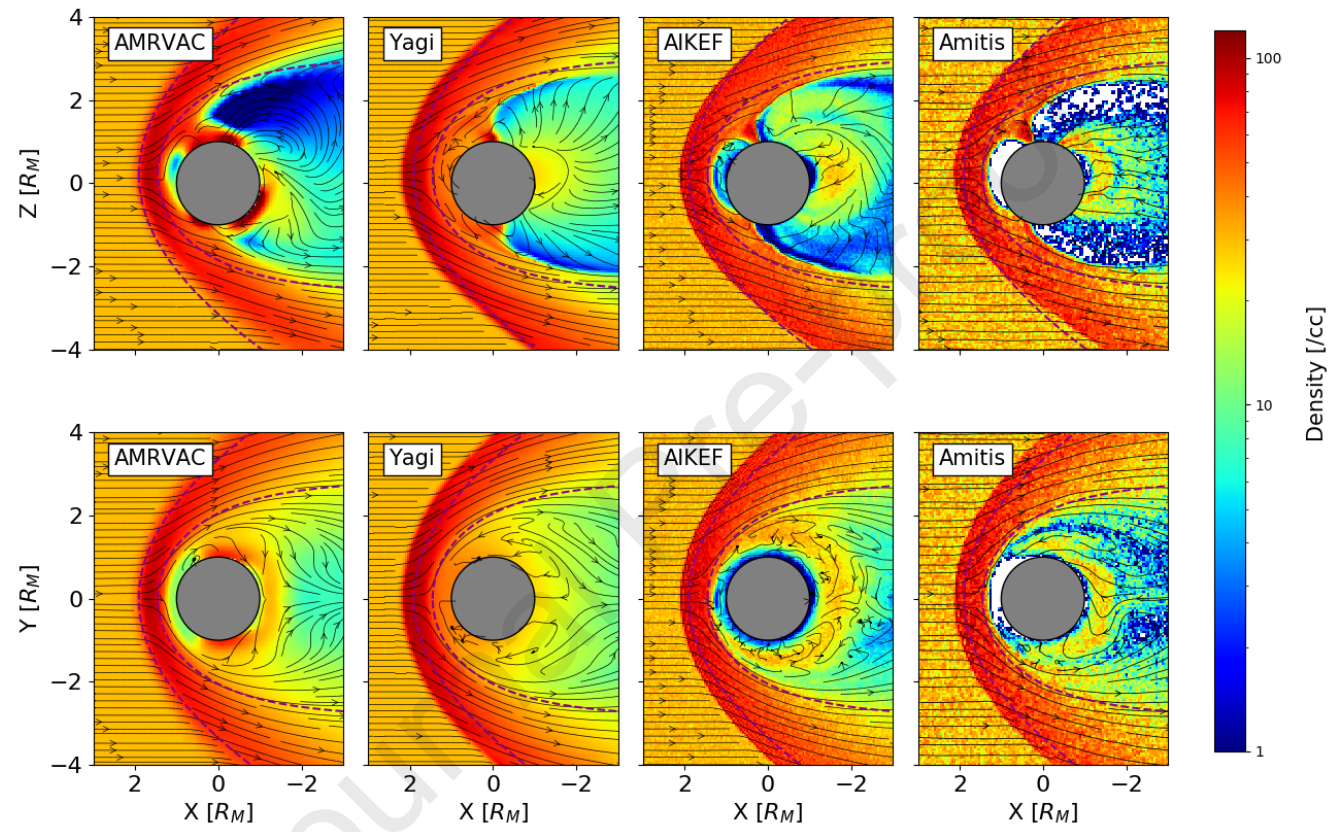

Figure 1: Maps of the solar wind proton density in the X-Z (top) and X-Y (bottom) plane at steady-state for the four simulation models under the northward IMF. The two panels on the left (right) are the results from the MHD (hybrid) codes. Length scales are normalized to the Mercury radius. Black arrows show the bulk velocity stream line and dashed two curves represent the averaged location of bowshock and magnetopause obtained by MESSENGER. [9] 
the dayside magnetosphere is not dilute and houses a similar density as the magnetosheath. The nightside structure is more symmetric and slightly tilted northward. The density concentration we find near the tail region in the AMRVAC model is not present here. The $X-Y$ cut of Yagi's model (bottom middle-left panel) also shows symmetric structure. Compared to AMRVAC, AMRVAC shows more detailed structure than that of Yagi's. Finally, only Yagi's model results show a north-south symmetric cusp region. The $X-$ $Z$ cut of AIKEF (upper middle-right panel), similar to Yagi and Amitis, produces a sharp bowshock and a more dilute dayside magnetosphere region. Both hybrid codes concur on narrower cusps as compared to the MHD models which visually identified. The structure in the nightside is tilted slightly northward, similar to Yagi's model. AIKEF in the $X-Y$ plane (bottom middle-right panel) show small asymmetric structure in the density, e.g., higher density inside the magnetosphere in the dawnside while lower density appears in the duskside.

Amitis does not employ any smoothing routines. Operating with a lower number of particles per cell as compared to AIKEF, the density maps in the $X-Z$ plane (upper right-most panel) therefore seem to contain more numerical noise. However, the bowshock and magnetopause are clearly captured and the solar wind plasma is denied from penetrating through the dayside magnetosphere as in the other simulations. The narrow cusp structure is similar to the results from AIKEF. The density configuration at the nightside of the planet seems to tend to northward. On the contrary to AIKEF, the $X-Y$ cut (bottom right-most panel) shows the opposite trend, e.g., the dayside magnetosphere seems to tend to dawnward while in the nightside 
there is denser plasma close to the surface.

In order to quantify the differences between the four models, Figure 2 presents the pressure profile along the subsolar line (X-axis). The dynamic and magnetic pressure are extracted and the locations of the bowshock and magnetopause have been identified. The four panels correspond to the four simulations. The solid lines show the dynamic (ram) pressure, $\varrho \mathbf{v}^{2}$ where $\varrho$ and $\mathbf{v}$ indicate the plasma density and the bulk velocity of the upstream solar wind, while the dashed lines represents the magnetic pressure, $B^{2} / 2 \mu_{0}$ where $B$ and $\mu_{0}$ denote the magnetic field magnitude and the vacuum permeability, respectively. The gray dash-dotted line is the magnetic pressure produced by an uncompressed dipole magnetic field with the same dipole moment as the models, providing information on how much the planetary magnetic field is compressed by the solar wind in each simulation run. The gray vertical line at $x=1.12 R_{\mathrm{M}}$ indicates the point where the dynamic pressure equals the uncompressed magnetic pressure and serves as a reference for the magnetopause location. For each model, the position of the bow shock (BS) has been identified as the maximum current density (red vertical solid line). The location of the magnetopause (MP; red vertical dashed line) has been identified in three different ways: (1) using the position of the most distant closed planetary field line that crosses the $\mathrm{X}$-axis, (2) using the position where the gas and magnetic pressure are equal, and (3) using the position where the current density has its maximum. All three methods were in excellent agreement for all four models (Table 3). 


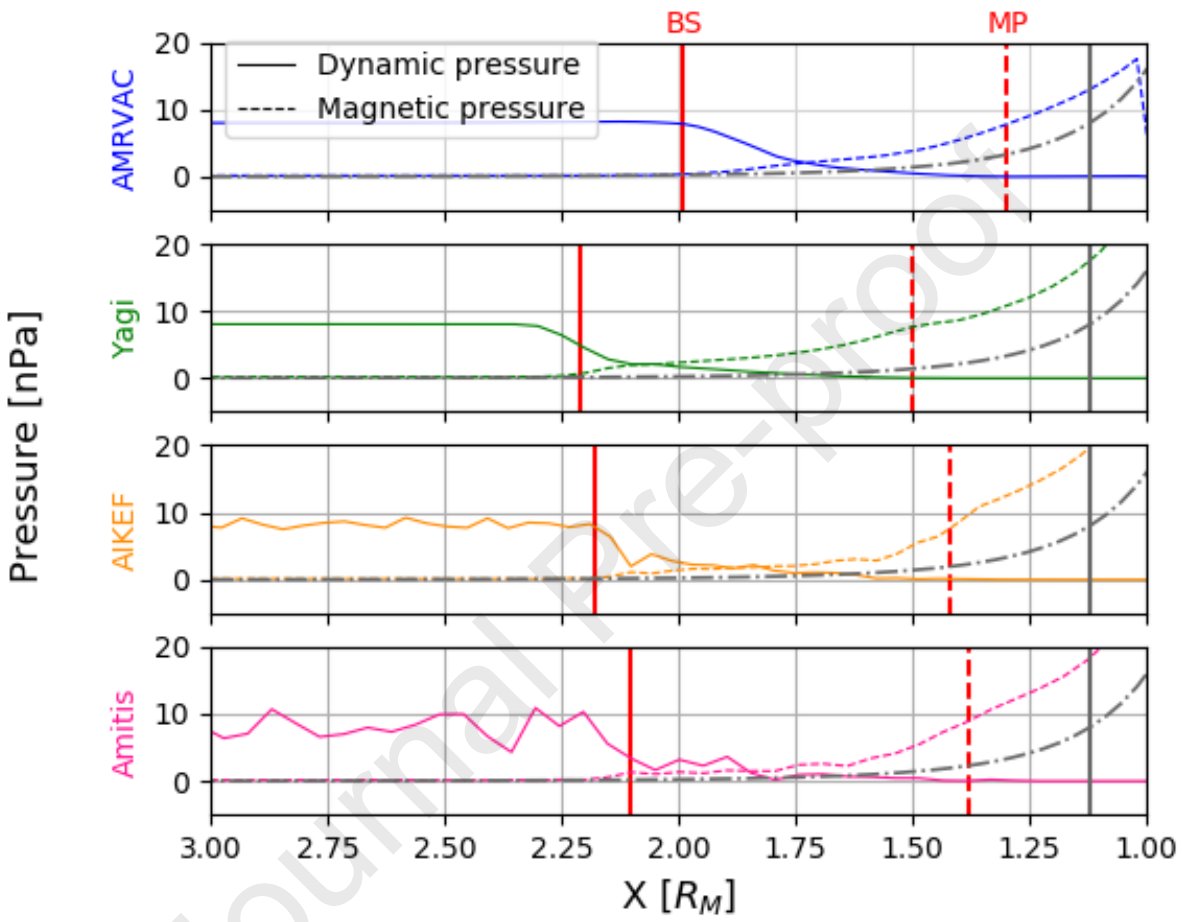

Figure 2: Dynamic (ram) and magnetic pressure profiles along the $\mathrm{X}$-axis (Sun-Mercury direction) from $3 R_{\mathrm{M}}$ upstream to the planet surface on the dayside for the four simulation codes. The results from the two MHD codes are shown on the top two panels and the hybrid model results on the two bottom panels. The gray dash-dotted line is the magnetic pressure produced by an uncompressed dipole magnetic field with the same dipole moment as the models. The positions of the bow shock (BS) and magnetopause (MP) are shown with red vertical solid and dashed lines. The grey vertical line gives the theoretical expected position of the magnetopause in the case of a radially diminishing magnetic field. 
Table 3: Bow shock and magnetopause locations at the subsolar point, and the thickness of the magnetosheath for the four simulation models.

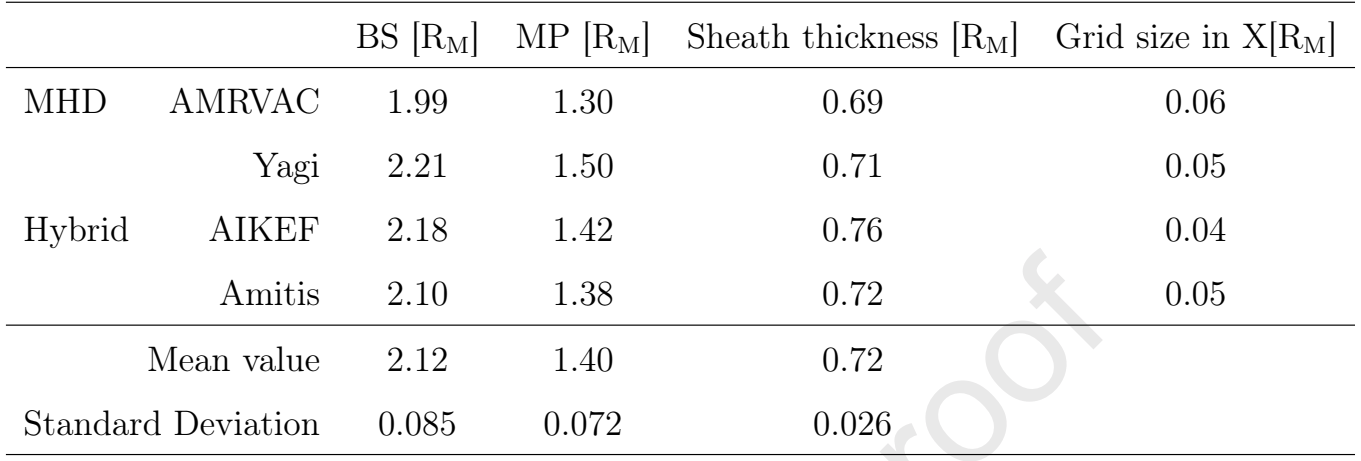

The locations of both the bow shock and magnetopause vary among the models with a range of $0.23 R_{\mathrm{M}}$ and $0.2 R_{\mathrm{M}}$, respectively (Figure 2 and Table 3). The mean position of the bow shock and magnetopause is found at $2.12 R_{\mathrm{M}}$, and $1.40 R_{\mathrm{M}}$, with a standard deviation of $\sigma=0.085$ and $\sigma=0.072$. The variation of the magnetopause location is greater than that of the bow shock as the former is more sensitive to the boundary condition applied at the surface and inside the planet. In an effort to eliminate the effects of the numerical implementation for comparison purposes, we compute the average thickness of the magnetosheath and find $0.72 R_{\mathrm{M}}$ with a standard deviation of 0.026. Thus, differences among models are small and within the uncertainty defined by the grid resolution. Note that the mean BS and MP locations simulated here with a purely northward IMF are within the ranges measured by MESSENGER [9].

In addition to the bow shock and magnetopause locations along the subsolar line, we further characterize the Hermean magnetosphere by means of 


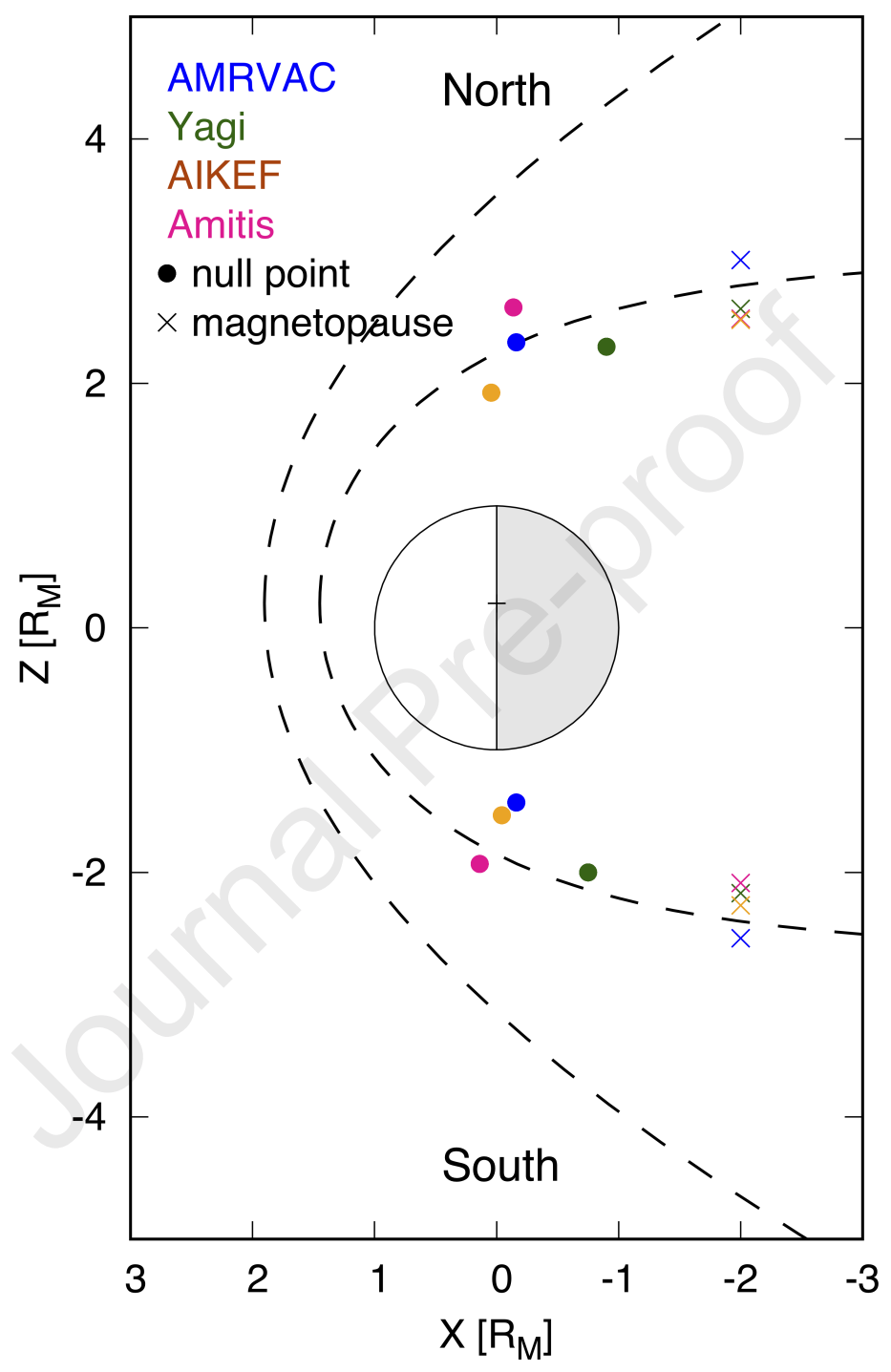

Figure 3: The locations of null points (colored circles) and magnetopause (colored crosses) in the X-Z plane. The dashed lines show the bow shock and magnetopause models defined by Slavin et al. [60] and Shue et al. [61], respectively. The magnetopause paraboloid has parameters $R_{s s}=1.45 R_{\mathrm{M}}$ and $\alpha=0.5$. The bow shock model parameters are $p=2.75 R_{\mathrm{M}}, \epsilon=1.04$, and $X_{0}=0.5 R_{\mathrm{M}}$. 

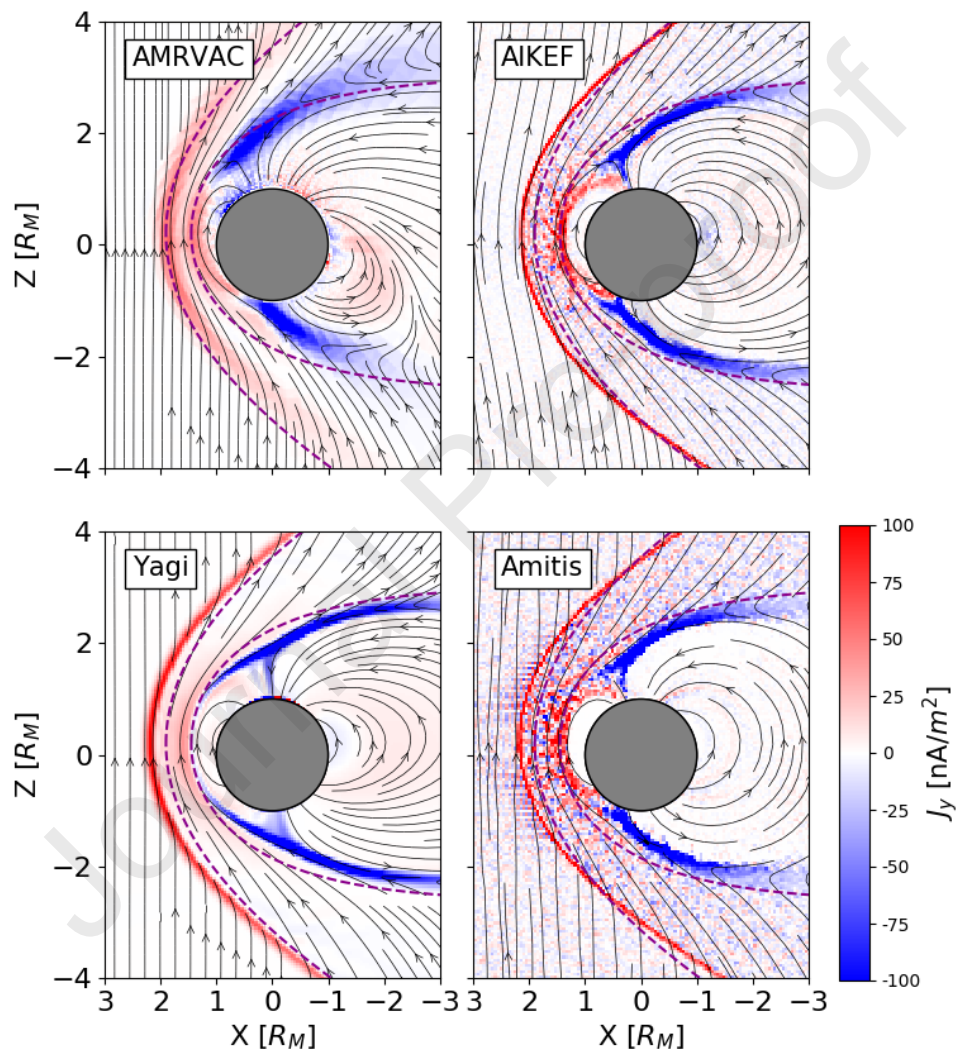

Figure 4: The y component of the current density in $X-Z$ plane. Black lines with arrow represent the magnetic field line and dashed purple lines are the empirical models of bowshock and magnetopause. 
the reconnection sites. Figure 3 shows a map in the $X-Z$ plane with its origin at the center of the planet. For reference, the two gray dashed lines represent the bow shock and magnetopause locations as predicted by the models of Slavin et al. [60], Shue et al. [61], Winslow et al. [9]. The positions of the reconnection sites, i.e. the null points, identified from the simulated magnetic field structure by our four models are indicated with full colored circles. The locations of the magnetopause at $X=-2 R_{M}$ are represented with colored crosses. On average, the null points near the northern cusp are located more towards the nightside, whereas the null point near the southern cusp are clustered more towards the terminator plane (with exception of Yagi's model). Due to the dipole offset towards the north, the southern null points are closest to the planet. Note that apart from the chosen plasma field conditions also various code-specific parameters, such as numerical resistivity, may play a role in where the reconnection sites develop. AMRVAC shows the largest north-south asymmetry on the location of null points, which is consistent with the tilted magnetic structure seen in Figure 1. The locations of the magnetopause on the nightside of the planet are obtained from the gradients of the density and the current density. With the exception of AMRVAC, these are located within the reference magnetopause model $\left(R_{s s}=1.45 R_{\mathrm{M}}\right.$ and $\alpha=0.5$ where $R_{s s}$ is the magnetopause distance at the subsolar and $\alpha$ is the flaring parameter $[61,9])$. Given the purely northward IMF, all four simulations can be considered in good agreement with the reference models.

Lastly in this section, the y-component of the current density in $X-$ $Z$ plane is presented in Figure 4. Since the IMF is purely northward in 
this case, we have highlighted only the $y$-component of the current density. Overall, 4 models show similar appearances, i.e., the current enhancement at the bowshock, positive current at the dayside magnetopause while negative current generated along the magnetopause in other region. AMRVAC shows the thicker layer at the bowshock and magnetopause, which is likely due to the lower grid resolution and Yagi's less diffusive numerical scheme, however, on the other hand, shock locations in dayside are in good agreement with the empirical models. Yagi's code shows sharp current structure than that of AMRVAC and since the magnetic field structure is more symmetric in the nightside, the current structure also shows more symmetric feature. Although both two hybrid codes show more busy figure because they treat ions as the particles, it is difficult to see the peak at the dayside magnetopause. Compared to Figure 1, since the density inside the dayside magnetosphere in AIKEF is low, those ions do not affect to the current system. 

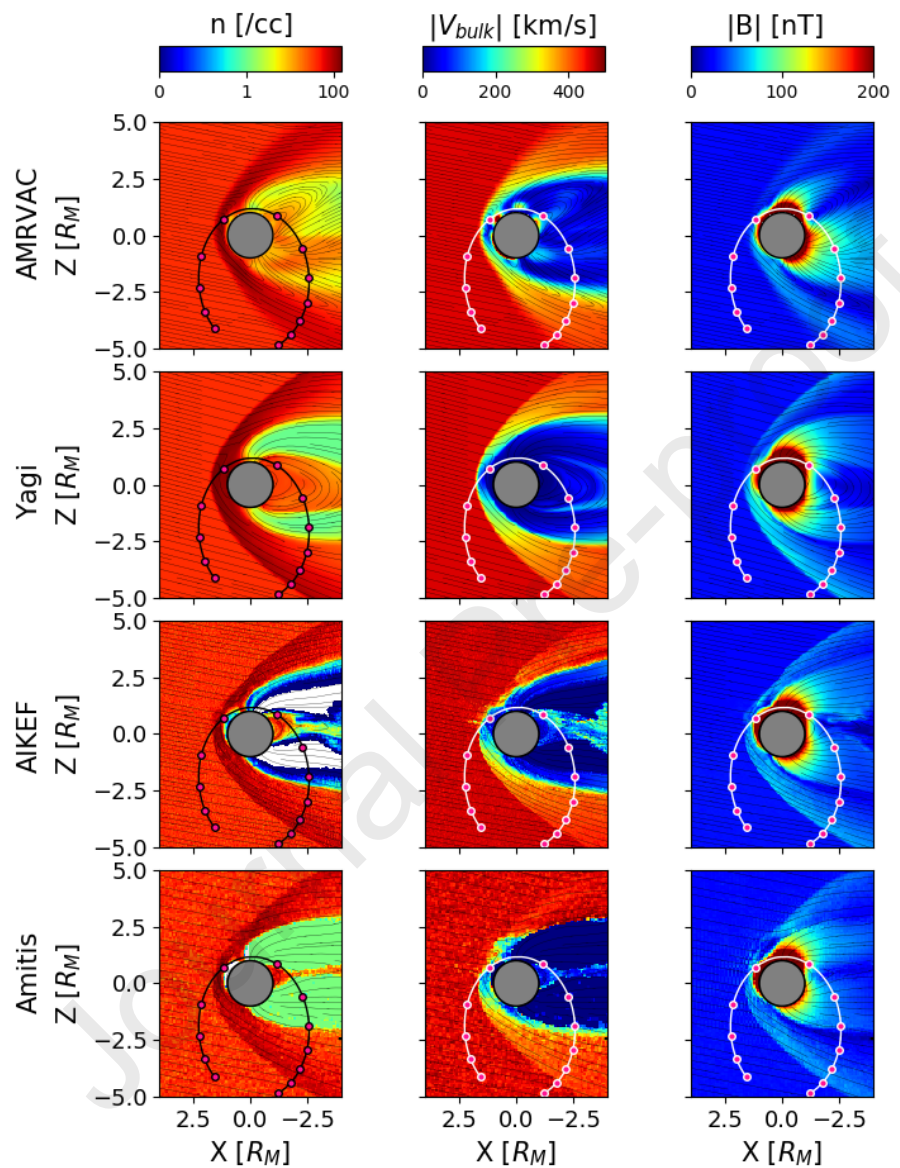

Figure 5: The overview of case $b$, from left to right, the panel shows the plasma density, bulk velocity, and magnitude of the magnetic field in $X-Z$ plane with magnetic field lines. MESSENGER trajectory is marked every $30 \mathrm{~min}$. In this orbit, MESSENGER was first crossing the boundaries in the southern hemisphere, moving into the nightside magnetosphere. 


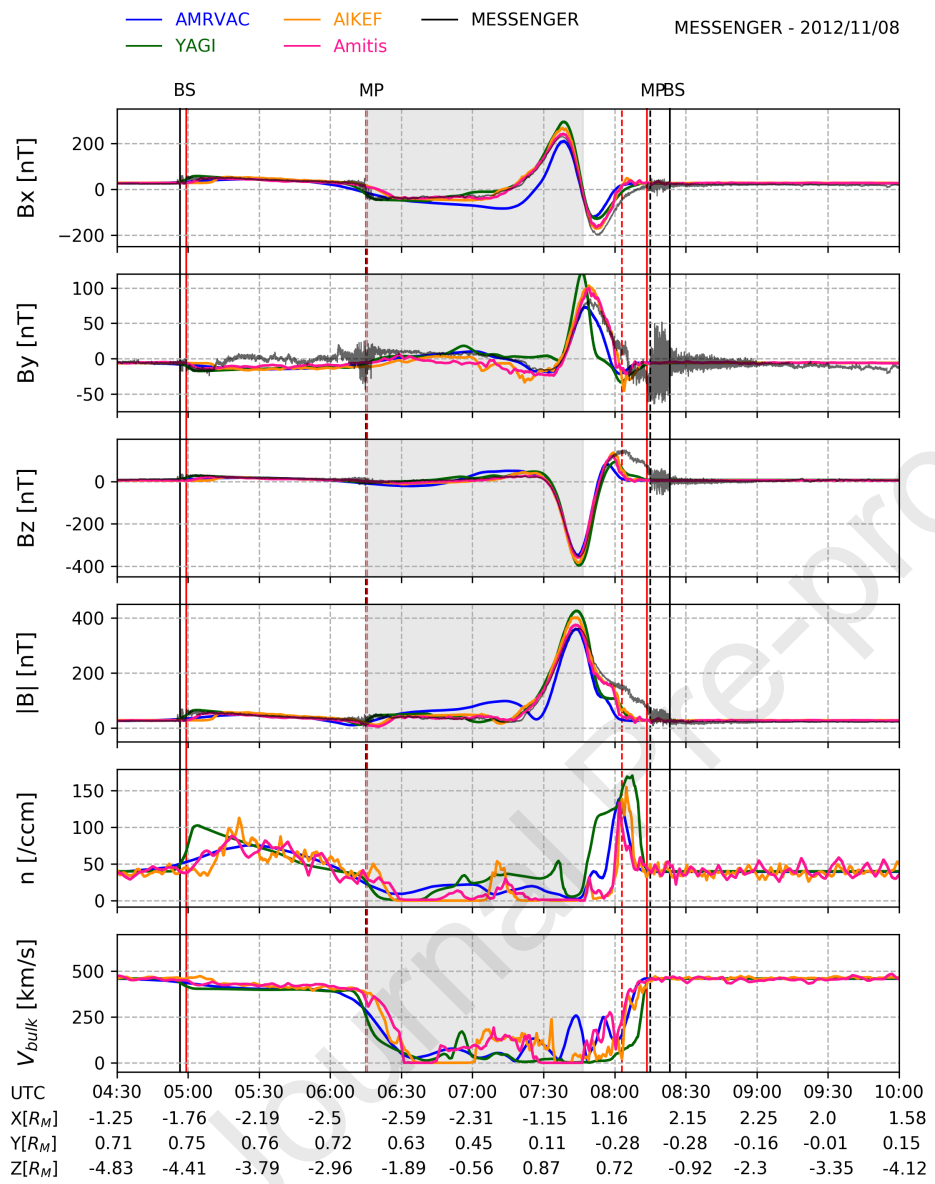

(b)

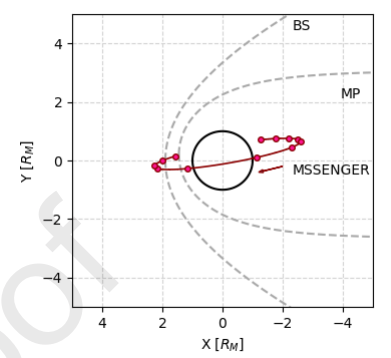

(c)

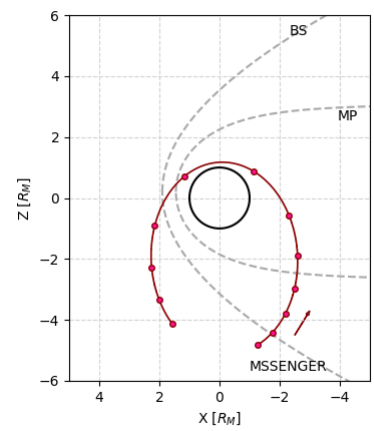

(a)

Figure 6: (a) Modeled and observed magnetic field and modeled particle data along orbit 1415 of MESSENGER. Blue, green, orange, and red lines shows the results from AMRVAC, Yagi, AIKEF and Amitis, respectively. The black lines shows the magnetometer data measured by MESSENGER. The bow shock (BS) and magnetopause (MP) crossings identified from the observations are indicated using a vertical solid and dashed black line. The averaged simulated shock and magnetopause crossings are shown by red solid and dashed lines, respectively. The nightside magnetosphere is indicated by a gray background. (b) and (c): the trajectory of MESSENGER spacecraft in $X-Y$ and $X-Z$ plane, respectively and the dots indicate 30 minute intervals of the MESSENGER trajectory. 
410

\subsection{Case b: MESSENGER comparison}

Figure 5 presents overview of the plasma density, the bulk velocity, and the magnetic field in $X-Z$ plane with the MESSENGER trajectory. All model results indicate similar features and magnitudes especially in the dayside magnetosphere. Interestingly, two hybrid models show the perturbed northern magnetopause. Figure 6 presents the magnetic field, density and plasma velocity profile along MESSENGER orbit 1415, on 8th of November, 2012, from 4:30 UT to 10:00 UT (see also section 3.2). The spacecraft entered the magnetosphere at the nightside along a south- to north-trajectory, roughly in the meridian plane with its closest approach near the magnetic north of Mercury, thus crossing the bow shock and magnetopause twice. Four vertical black lines indicate the bow shock and magnetopause locations for both the inbound and outbound crossings observed by MESSENGER. Superimposed on their respective panels are the simulated profiles from Case $a$ along the same trajectory. The red vertical lines are the averaged simulated shock crossings from the four models. Overall, our models are in close agreement with the MESSENGER data for the inbound part of the orbit. With time differences of up to $12 \mathrm{~min}$, equal to $\sim 0.28 \mathrm{R}_{\mathrm{M}}$ along the spacecraft trajectory for the inbound orbit, and $17 \mathrm{~min}\left(\sim 1.1 \mathrm{R}_{\mathrm{M}}\right)$ for the outbound orbit, the locations of the BS and MP agree less well for the outbound section of the MESSENGER orbit. The BS locations match better for the inbound part of the orbit.

The variations of the magnetic field along the trajectory seem to be in good agreement among codes and with MESSENGER data, while more per- 
turbed variations in density and bulk velocity profiles. The small fluctuations in two hybrid are results of particle noises.

Since we used the inbound part of the orbit to constrain the upstream plasma parameters to initialize the simulations, it is possible that variations in the solar wind dynamic pressure during the orbit may be responsible for the larger discrepancy in the outbound part of the orbit. Interestingly, there are distinct differences between the MHD and the hybrid codes (panel 4 of Figure 6). Around 7:50 UT, when the spacecraft is inside the dayside magnetosheath, the results from the two hybrid simulations are in agreement with the observations, while both MHD models predict a lower magnetic field intensity. The spacecraft passes through the northern cusp and then moves towards the dayside region. At 8:10 UT, near the peak density, we find the compressed magnetosheath plasma. As expected, also the density and velocity profiles predicted by the hybrid codes show steeper profiles at the shock crossing compared to the MHD models.

\section{Discussion}

\subsection{Case b: MESSENGER comparison}

Due to the limited particle instrumentation on board the single-spacecraft MESSENGER mission, it is not possible to constrain the exact solar wind plasma parameters to set up a comparison simulation. More precisely, because the particle instrument onboard MESSENGER was protected by the sun shield and thus it had a limited field of view, this condition prevented the instrument from observing the majority of solar wind. Therefore, density and 
velocity, temperature of solar wind cannot be derived and those parameters must be assumed when input parameters were considered [62] In addition, the solar wind varies in time and may even be changing significantly while MESSENGER crosses the magnetosphere. This may be the reason for some of the discrepancies between simulated and measured profiles. In Figure 7 we compute the difference between the observed and modeled magnetic fields along our chosen MESSENGER orbit. We exclude the time frame between the two MP crossings (from 6:15 UT to 7:45 UT) while MESSENGER passed the nightside region of the magnetosphere (indicated by a gray background), to focus on the cusp and boundary layer locations.

Just before 5:00 UT, MESSENGER crosses the BS for the first time, about 3 min before the predicted averaged time stamp from our models. Continuing along the trajectory, both the modeled X- and Z-component agree well with MESSENGER, whereas the models underestimate the magnetic field contribution by roughly $15 \mathrm{nT}$ along the $\mathrm{Y}$ direction. Our models agree on the inbound MP crossing time at 6:15 UT within a 5 min time range. At 7:50 UT, when MESSENGER moves from the nightside to the dayside in the northern hemisphere, all four models predict a magnetic pile-up, although none of the models fully reproduces the fine-scale structure. Immediately following the pile-up, the models underestimate the magnetic field strength by roughly $100 \mathrm{nT}$. Note that the different profiles in Figure 6 show no real 'winner' among the four simulation models as all codes show different smallscale discrepancies with the MESSENGER measurements at the boundaries between the distinct plasma regions of Mercury's magnetosphere. It shows 
the need for a multi-spacecraft mission, such as BepiColombo, to simultaneously measure the local Hermean and upstream plasma environment in order to fine-tune the inputs to numerical models and in turn characterize Mercury's global plasma environment.

\subsection{Virtual sampling along Mio/MPO orbits}

One of the major goals of the SHOTS project, next to comparing different simulation approaches with available MESSENGER measurements, is to help prepare the community for the forthcoming BepiColombo mission by making available a catalog of simulation that showcases the plasma structure and dynamics of Mercury's magnetosphere under different solar wind conditions. In the first step of this project, we chose four different (MHD and hybrid) simulation models. Figures 8 and 9 show the plasma characteristics expected along a typical Mio and MPO orbit, respectively, during the planned nominal orbital phase of BepiColombo. We chose the upstream solar wind conditions identical to the MESSENGER case studied above (Case $b$ in Table 2). For ease of comparison, the orbital plane is set perpendicular to the $\mathrm{X}$ direction, corresponding to BepiColombo's operations planned for April 4, 2026. During this period, both Mio and MPO remain inside the magnetosphere and the plasma environment will be fully observed by the magnetometers on board Mio and MPO [63, 64], the Mercury Plasma Particle Experiment (MPPE) [65] and the Plasma Wave Investigation (PWI) [66] onboard Mio, and the Search for Exospheric Refilling and Emitted Natural Abundances (SERENA) on board MPO [67]. 
MESSENGER - 2012/11/08

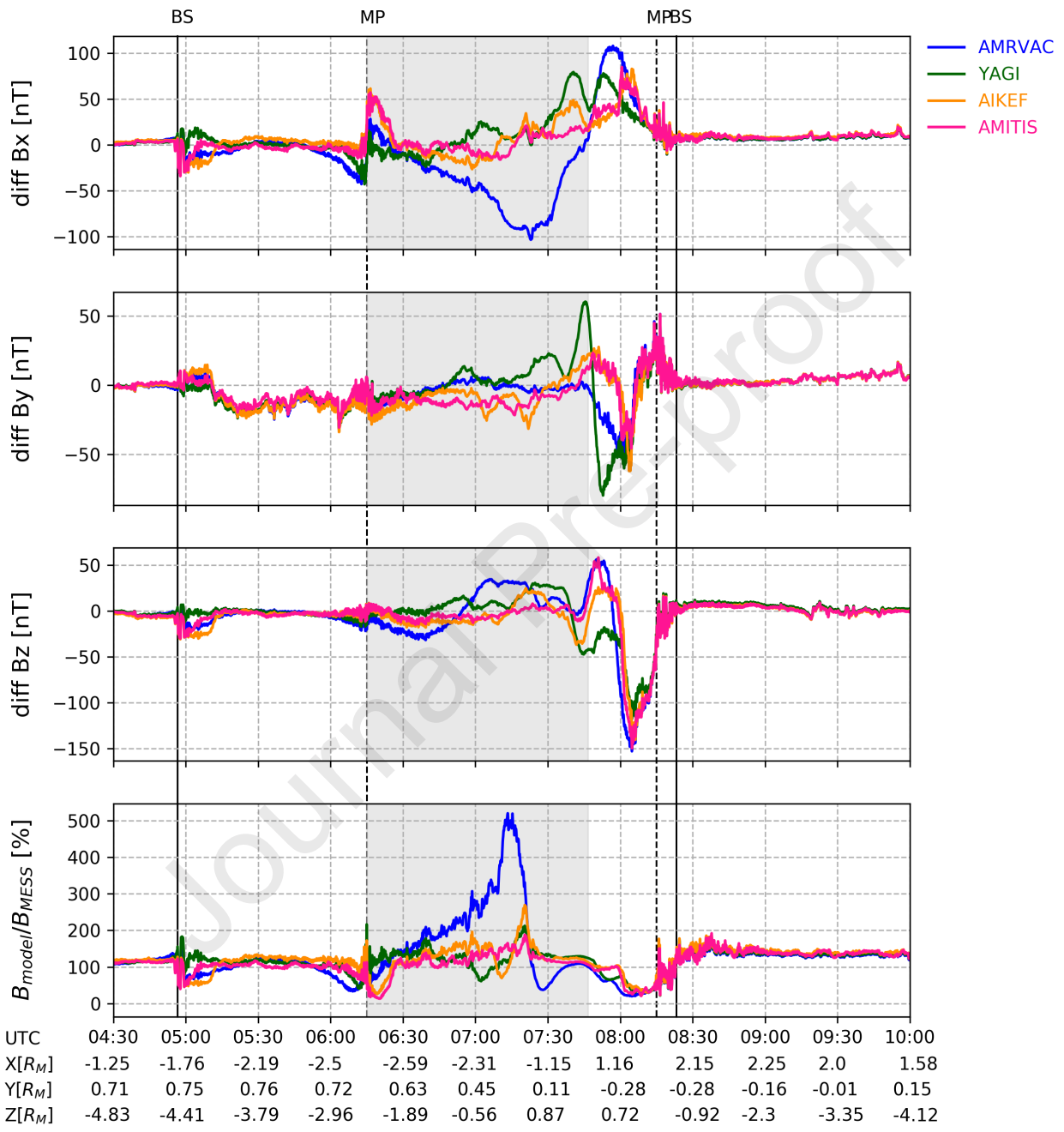

Figure 7: Difference between the simulation results and the MESSENGER magnetic field measurements ( $\left.B_{\text {model }}-B_{M E S S}\right)$. The bow shock (BS) and magnetopause (MP) crossings identified from the observations are indicated with a vertical solid and dashed line, respectively and the region MESSENGER passing through inside the nightside magnetosphere is indicated by a gray background. 
In both Figures 8 and 9 the solid vertical lines indicate the southern $\left(\mathrm{T}_{\mathrm{S}}\right)$ and northern $\left(\mathrm{T}_{\mathrm{N}}\right)$ terminator crossing and the dashed vertical line is the time when the spacecraft crosses the sub-solar point (SSP). During this period, Mio will be moving along the frontside magnetopause (from 15:15 UT to 15:35 UT) where our models predict an enhancement of the magnetic field intensity and plasma velocity. Note that the predicted profiles closest to the planet are not in agreement, possibly due to the numerical treatment of the planetary boundary condition (see also Figure 1). Next, Mio will move across the cusp region and re-enter the nightside magnetosphere from the north. Large discrepancies among models around the subsolar point to the northern terminator can be explained by the orbit of the Mio spacecraft. Since Mio is moving along the magnetopause, different locations of the magnetopause in each model are critical. Mio is clearly inside the magnetopause in AIKEF and Amitis around the subsolar point but probably not in Yagi and AMRVAC. After crossing subsolar point around 15:17 UT, the density and velocity profiles diverge. A similar trend is not visible in the magnetic field predictions. This may be the region where the ion dynamics has the largest impact, i.e., the finite Larmor radius effect of particle must be taken into account in the magnetosheath, hence producing the largest differences between the hybrid and MHD models.

MPO will orbit significantly closer to the planet. The spacecraft will leave the southern magnetosphere via the nightside, then crosses the terminator and enters the dayside magnetosphere. Just before 15:00 UT and around 15:35 UT, the two hybrid models and AMRVAC predict a significant density 
enhancement, indicating the presence of trapped particles while Yagi show significantly different appearance. In Yagi's model, the dayside magnetosphere is not clearly seen (see Figure 5), and thus, the spacecraft seems to observe rather the magnetosheath component than the component inside the magnetopause at the time between 14:30 UT to 14:50 UT. The spacecraft passes through the cusp region (light blue areas in Figure 9). MPO's trajectory will be most useful to better understand the structure and dynamics of the magnetosphere closest to the planet and in particular near the southern hemisphere. This region has been explored less due to MESSENGER's highly inclined and eccentric orbit.

Sampling the virtual orbits of Mio and MPO for a variety of solar wind parameters and/or models, we will be able to predict when the spacecraft most likely cross the cusp, the plasma sheet and the shocked regions within certain margins. These margins depend on the characteristics of the simulation and the physical processes included in the model. The combined measurements from the two spacecraft will provide a wealth of information on the Hermean plasma environment, however, to reproduce adequately the environment in 3D, numerical 3D models are essential. Thus, making predictive simulations with the information by combined spacecraft measurements a necessity to maximize the scientific return of the mission. To this effect, the simulation domain needs to be extended farther downstream of the planet to capture better the magnetotail region. Also temporal information needs to be included. In this study, our hybrid models have not implemented its tenuous exosphere based on the previous works [23, 33]that shows Mercury's 
exosphere is tenuous enough to not significantly affect on Mercury's magnetospheric system. Our first goal was to purely compare between MHD and hybrid simulations so that we see the ion kinetics. However revealing the role of the exosphere is also the one of the key questions of Mercury's science. It will be investigated further in near future. Finally, also Particle-In-Cell and Vlasov simulations that include the electron dynamics are needed.

\section{Conclusions}

We have compared the results of four different (MHD and hybrid) global simulation models with the same input parameters to simulate the solar wind interaction with Mercury's dayside magnetosphere. Because the effect of the existence of its tenuous exosphere is small to affect the structure of Mercury's magnetosphere, the exosphere has not been implemented in our models. All our models produced a similar global structure in the dayside consistent with empirical (analytical) fits for the locations of the bow shock and the magnetopause. In contrast to the dayside magnetosphere, although it is out of our scope, the magnetic field configuration in the nightside magnetosphere differs among models, and the plasma distribution and bulk velocity close to the surface are also different. The significant difference between MHD and hybrid models is not seen in this study, thus, the differences between the simulations predictions seem to be caused numerically, i.e., the planetary boundary conditions, different size of the grid, numerical scheme, rather than the self-consistent ion kinetics included in the model. 


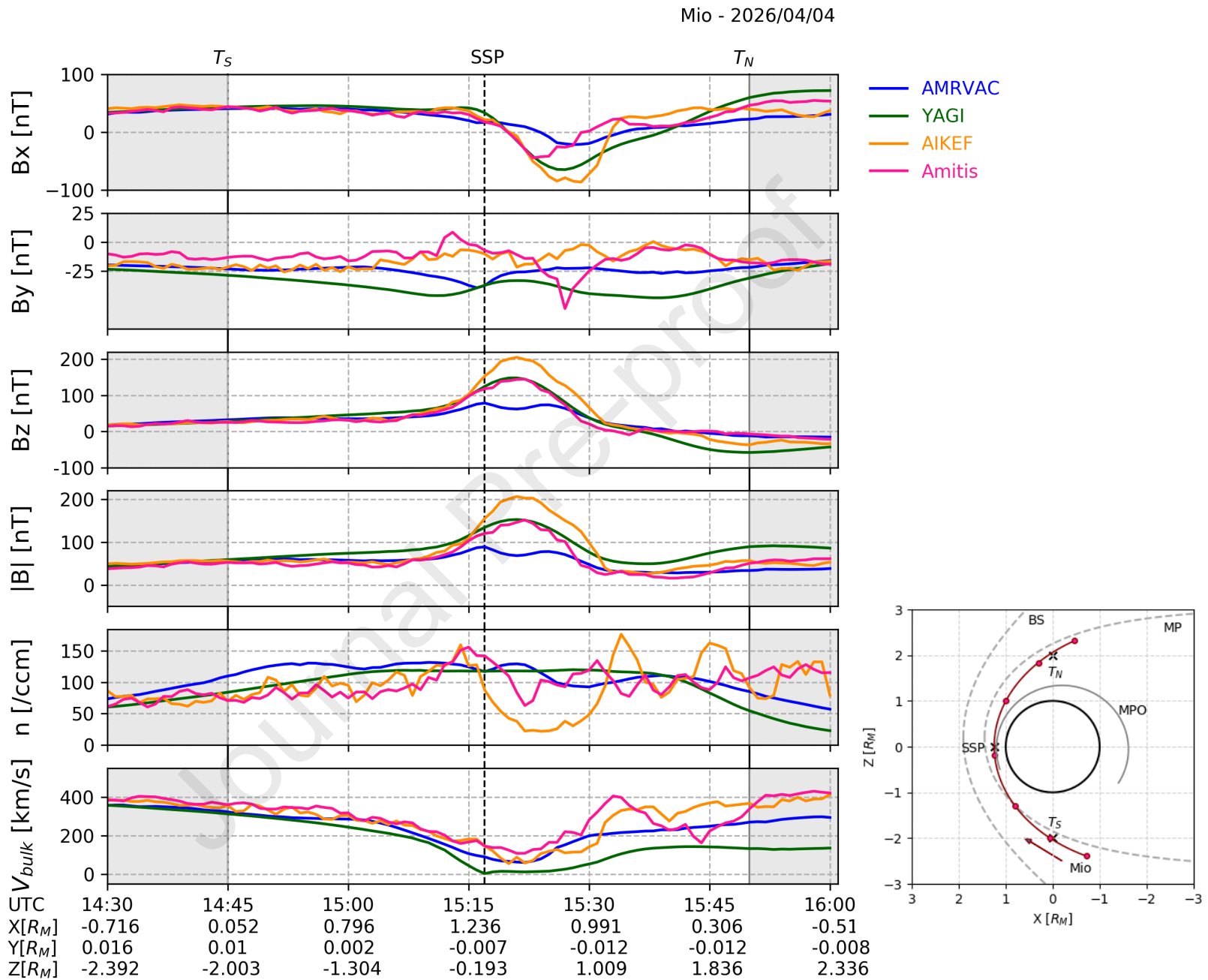

Figure 8: Magnetic field and particle data along a representative Mio orbit. The red dots along the trajectory in the inset panel show the position of the spacecraft along $15 \mathrm{~min}$ intervals. The solid vertical lines indicate the crossing of the southern $\left(\mathrm{T}_{\mathrm{S}}\right)$ and northern $\left(\mathrm{T}_{\mathrm{N}}\right)$ terminator, the dashed vertical line is the time when the spacecraft crosses the subsolar point (SSP). The nightside region $(X<0)$ is indicated by a gray background. 


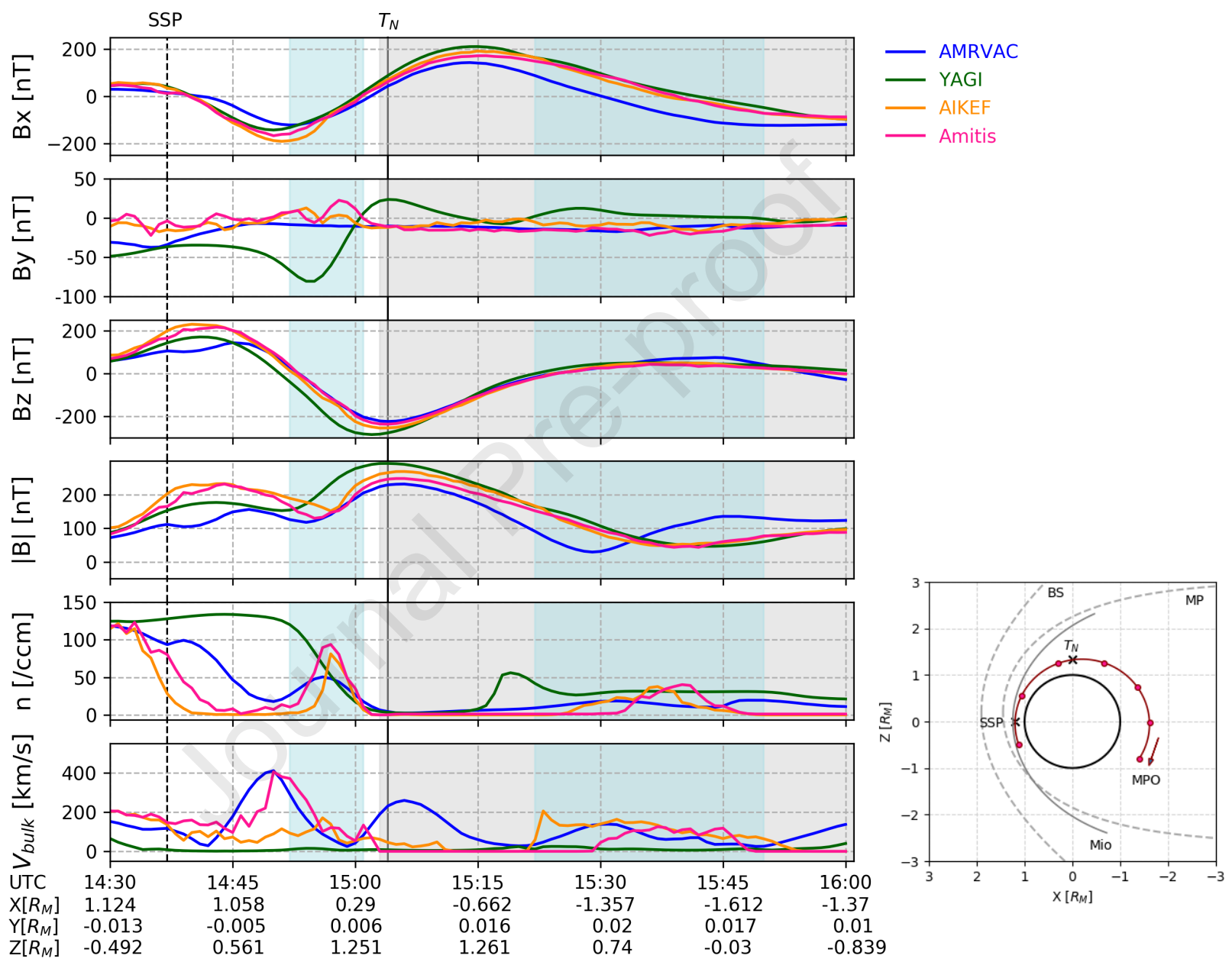

Figure 9: Magnetic field and particle data along a representative MPO orbit. The red dots along the trajectory in the inset panel show the position of the spacecraft along $15 \mathrm{~min}$ intervals. The solid vertical lines indicate northern $\left(\mathrm{T}_{\mathrm{N}}\right)$ terminator and the dashed vertical line is the time when the spacecraft crosses the subsolar point (SSP). The nightside region $(X<0)$ is indicated by a gray background. 
571

We cross-compared our results with a theoretical northward IMF scenario (Case $a$ ) and MESSENGER orbit 1415 (Case $b$ ), indicated to have stable solar wind conditions with a northward IMF similar to the theoretical case. In Case a, the standard deviations for the predicted mean locations of the bow shock, magnetopause and the thickness of magnetosheath are small. For Case b, during the inbound part of the orbit, time differences for the bow shock and the magnetopause crossings are found up to 12 minutes, corresponding to a distance along the orbit of $0.28 \mathrm{R}_{\mathrm{M}}$. For the outbound section of the orbit, the maximum time difference increased to 17 minutes, equivalent to $1.1 \mathrm{R}_{\mathrm{M}}$.

One of the major goals of SHOTS is to prepare a catalog of simulations that can predict the plasma environment in- and outside the Hermean magnetosphere under different solar wind conditions, in this way contributing to maximizing the scientific return of the forthcoming BepiColombo observations. Here, for the first time, we have extracted the data from our models along representative Mio and MPO orbits. A long-lasting project-based and community-wide effort will be important both for the forthcoming BepiColombo's Mercury flybys and during its nominal orbital phase.

\section{Acknowledgments}

The authors acknowledge support from the French space plasma physics data centre (Centre de Données de la Physique des Plasmas, CDPP: http:// www. cdpp. eu funded by CNES and CNRS). J.D. acknowledges support from 
NASA's Solar System Exploration Research Virtual Institute (SSERVI): Institute for Modeling Plasmas, Atmosphere, and Cosmic Dust (IMPACT), and the NASA High-End Computing (HEC) Program through the NASA Advanced Supercomputing (NAS) Division at Ames Research Center. D.H. was supported by the German Ministerium für Wirtschaft und Energie and the German Zentrum für Luft-und Raumfahrt under contract 50 QW 1501. W.E. was supported by DFG (German Research Foundation) under contract HE8016/1-1. S.F. acknowledges support from Swedish National Research Council, grant \#2018- 03454, Swedish National Space Agency, grants \#2018-C and \#2018-N. Amitis simulations conducted using computational resources provided by the Swedish National Infrastructure for Computing (SNIC), projects SNIC2019/3-178 and SNIC2020-5-101 at the High Performance Computing Center North (HPC2N), Umeå University, Sweden. French co-authors would like to acknowledge the support of CNES for the BepiColombo mission. Part of this work has been done in the framework of the Sun Planet Interactions Digital Environment on Request (SPIDER) activities of the Europlanet 2024 RI project. Europlanet 2024 RI has received funding from the European Union's Horizon 2020 research and innovation programme under grant agreement No 871149 .

All data necessary to validate the findings presented in this manuscript can be found at https://github.com/jandeca/Aizawa_etal_PSS_2020 (DOI: to be provided) in accordance with the Findability Accessibility, Interoperability, and Reuse (FAIR) principles. The magnetometer data from the MESSENGER observations are available in the Planetary Data System (https: 
610

611

//pds-ppi .igpp.ucla.edu).

[1] N. F. Ness, K. W. Behannon, R. P. Lepping, Y. C. Whang, The magnetic field of Mercury, Journal of Geophysical Research (1896-1977) 80 (1975) 2708-2716. doi:10.1029/JA080i019p02708.

[2] Y. C. Whang, Magnetospheric magnetic field of Mercury, Journal of Geophysical Research (1896-1977) 82 (1977) 1024-1030. doi:10.1029/ JA082i007p01024.

[3] H. Korth, B. J. Anderson, C. L. Johnson, J. A. Slavin, J. M. Raines, T. H. Zurbuchen, Structure and configuration of Mercury's magnetosphere, in: S. C. Solomon, L. R. Nittler, B. J. Anderson (Eds.), Mercury: The View after MESSENGER, Cambridge University Press, 2018, pp. 430-460.

[4] J. A. Slavin, D. N. Baker, D. J. Gershman, G. C. Ho, S. M. Imber, S. M. Krimigis, T. Sundberg, Mercury's dynamic magnetosphere, in: S. C. Solomon, L. R. Nittler, B. J. Anderson (Eds.), Mercury: The View after MESSENGER, Cambridge University Press, 2018, pp. 461-496.

[5] E. Jang, J. T. Zhao, C. Yue, Q. G. Zong, Y. Liu, Z. Y. Liu, Energetic Ion Dynamics Near the Cusp Region of Mercury, The Astrophysical Journal 892 (2020) 10. doi:10.3847/1538-4357/ab74d1.

[6] J. A. Slavin, M. H. Acuña, B. J. Anderson, D. N. Baker, M. Benna, G. Gloeckler, R. E. Gold, G. C. Ho, R. M. Killen, H. Korth, S. M. Krimigis, R. L. McNutt, L. R. Nittler, J. M. Raines, D. Schriver, S. C. 
Solomon, R. D. Starr, P. Trávníček, T. H. Zurbuchen, Mercury's Magnetosphere After MESSENGER's First Flyby, Science 321 (2008) 85-89. doi:10.1126/science. 1159040.

[7] J. A. Slavin, M. H. Acuña, B. J. Anderson, D. N. Baker, M. Benna, S. A. Boardsen, G. Gloeckler, R. E. Gold, G. C. Ho, H. Korth, S. M. Krimigis, R. L. McNutt, J. M. Raines, M. Sarantos, D. Schriver, S. C. Solomon, P. Trávníček, T. H. Zurbuchen, Messenger observations of magnetic reconnection in Mercury's magnetosphere, Science 324 (2009) 606-610. doi:10.1126/science.1172011.

[8] J. A. Slavin, B. J. Anderson, D. N. Baker, M. Benna, S. A. Boardsen, G. Gloeckler, R. E. Gold, G. C. Ho, H. Korth, S. M. Krimigis, R. L. McNutt, L. R. Nittler, J. M. Raines, M. Sarantos, D. Schriver, S. C. Solomon, R. D. Starr, P. M. Trávníček, T. H. Zurbuchen, Messenger observations of extreme loading and unloading of Mercury's magnetic tail, Science 329 (2010) 665-668. doi:10.1126/science.1188067.

[9] R. M. Winslow, B. J. Anderson, C. L. Johnson, J. A. Slavin, H. Korth, M. E. Purucker, D. N. Baker, S. C. Solomon, Mercury's magnetopause and bow shock from MESSENGER Magnetometer observations, Journal of Geophysical Research (Space Physics) 118 (2013) 2213-2227. doi:10. 1002/jgra. 50237.

[10] J. A. Slavin, G. A. DiBraccio, D. J. Gershman, S. M. Imber, G. K. Poh, J. M. Raines, T. H. Zurbuchen, X. Jia, D. N. Baker, K.-H. Glassmeier, S. A. Livi, S. A. Boardsen, T. A. Cassidy, M. Sarantos, T. Sundberg, A. Masters, C. L. Johnson, R. M. Winslow, B. J. Anderson, H. Korth, 
R. L. McNutt Jr., S. C. Solomon, Messenger observations of Mercury's dayside magnetosphere under extreme solar wind conditions, Journal of Geophysical Research: Space Physics 119 (2014) 8087-8116. doi:10. 1002/2014JA020319.

[11] X. Jia, J. A. Slavin, G. Poh, G. A. DiBraccio, G. Toth, Y. Chen, J. M. Raines, T. I. Gombosi, Messenger observations and global simulations of highly compressed magnetosphere events at Mercury, Journal of Geophysical Research: Space Physics 124 (2019) 229247. URL: https://agupubs.onlinelibrary.wiley.com/doi/abs/ 10.1029/2018JA026166. doi:10.1029/2018JA026166.

[12] J. A. Slavin, H. R. Middleton, J. M. Raines, X. Jia, J. Zhong, W.-J. Sun, S. Livi, S. M. Imber, G.-K. Poh, M. Akhavan-Tafti, J. M. Jasinski, G. A. DiBraccio, C. Dong, R. M. Dewey, M. L. Mays, Messenger observations of disappearing dayside magnetosphere events at Mercury, Journal of Geophysical Research: Space Physics 124 (2019) 6613-6635. doi:10. 1029/2019JA026892.

[13] R. M. Winslow, N. Lugaz, L. Philpott, C. J. Farrugia, C. L. Johnson, B. J. Anderson, C. S. Paty, N. A. Schwadron, M. A. Asad, Observations of Extreme ICME Ram Pressure Compressing Mercury's Dayside Magnetosphere to the Surface, The Astrophysical Journal 889 (2020) 184. doi:10.3847/1538-4357/ab6170. arXiv: 1903.00577.

[14] E. Kallio, P. Wurz, R. Killen, S. McKenna-Lawlor, A. Milillo, A. Mura, S. Massetti, S. Orsini, H. Lammer, P. Janhunen, On the impact of multiply charged heavy solar wind ions on the surface of Mercury, the 
Moon and Ceres, Planetary and Space Science 56 (2008) 1506-1516. doi:10.1016/j.pss.2008.07.018.

[15] J. M. Raines, D. J. Gershman, J. A. Slavin, T. H. Zurbuchen, H. Korth, B. J. Anderson, S. C. Solomon, Structure and dynamics of Mercury's magnetospheric cusp: MESSENGER measurements of protons and planetary ions, Journal of Geophysical Research (Space Physics) 119 (2014) 6587-6602. doi:10.1002/2014JA020120.

[16] S. Fatemi, A. R. Poppe, S. Barabash, Hybrid simulations of solar wind proton precipitation to the surface of Mercury, Journal of Geophysical Research: Space Physics 125 (2020) e2019JA027706. doi:10.1029/2019JA027706.

[17] X. Jia, J. A. Slavin, T. I. Gombosi, L. K. S. Daldorff, G. Toth, B. van der Holst, Global mhd simulations of Mercury's magnetosphere with coupled planetary interior: Induction effect of the planetary conducting core on the global interaction, Journal of Geophysical Research: Space Physics 120 (2015) 4763-4775. doi:10.1002/2015JA021143.

[18] C. L. Johnson, L. C. Philpott, B. J. Anderson, H. Korth, S. A. Hauck, D. Heyner, R. J. Phillips, R. M. Winslow, S. C. Solomon, MESSENGER observations of induced magnetic fields in Mercury's core, Geophysical Research Letters 43 (2016) 2436-2444. doi:10.1002/2015GL067370.

[19] K. Kabin, T. Gombosi, D. DeZeeuw, K. Powell, Interaction of Mercury with the solar wind, Icarus 143 (2000) 397 - 406. doi:http://dx.doi. org/10.1006/icar.1999.6252. 
[20] M. Benna, B. J. Anderson, D. N. Baker, S. A. Boardsen, G. Gloeckler, R. E. Gold, G. C. Ho, R. M. Killen, H. Korth, S. M. Krimigis, M. E. Purucker, R. L. McNutt, J. M. Raines, W. E. McClintock, M. Sarantos, J. A. Slavin, S. C. Solomon, T. H. Zurbuchen, Modeling of the magnetosphere of Mercury at the time of the first MESSENGER flyby, Icarus 209 (2010) 3-10. doi:10.1016/j.icarus.2009.11.036.

[21] C. Dong, L. Wang, A. Hakim, A. Bhattacharjee, J. A. Slavin, G. A. DiBraccio, K. Germaschewski, Global Ten-Moment Multifluid Simulations of the Solar Wind Interaction with Mercury: From the Planetary Conducting Core to the Dynamic Magnetosphere, Geophysical Research Letters 46 (2019) 11,584-11,596. doi:10.1029/2019GL083180. arXiv: 1904.02695.

[22] E. Kallio, P. Janhunen, Modelling the solar wind interaction with Mercury by a quasi-neutral hybrid model, Annales Geophysicae 21 (2003) 2133-2145. doi:10.5194/angeo-21-2133-2003.

[23] E. Kallio, P. Janhunen, The response of the hermean magnetosphere to the interplanetary magnetic field, Advances in Space Research 33 (2004) 2176 - 2181. URL: http://www. sciencedirect.com/science/article/pii/S0273117703004472. doi:https://doi.org/10.1016/S0273-1177(03)00447-2, mercury, Mars and Saturn.

[24] P. Trávníček, P. Hellinger, D. Schriver, Structure of Mercury's magnetosphere for different pressure of the solar wind: Three dimensional 
hybrid simulations, Geophysical Research Letters 34 (2007) 5104. doi:10 . 1029/2006GL028518.

[25] P. M. Trávníček, D. Schriver, P. Hellinger, D. Herčík, B. J. Anderson, M. Sarantos, J. A. Slavin, Mercury's magnetosphere: solar wind interaction for northward and southward interplanetary magnetic field: Hybrid simulation results, Icarus 209 (2010) 11 - 22 . doi:http://dx.doi.org/10.1016/j.icarus.2010.01.008.

[26] Y.-C. Wang, J. Mueller, U. Motschmann, W.-H. Ip, A hybrid simulation of Mercury's magnetosphere for the MESSENGER encounters in year 2008, Icarus 209 (2010) 46-52. doi:10.1016/j .icarus.2010.05.020.

[27] E. Richer, R. Modolo, G. M. Chanteur, S. Hess, F. Leblanc, A global hybrid model for Mercury's interaction with the solar wind: Case study of the dipole representation, Journal of Geophysical Research (Space Physics) 117 (2012) A10228. doi:10.1029/2012JA017898.

[28] D. Herčík, P. M. Trávníček, J. R. Johnson, E.-H. Kim, P. Hellinger, Mirror mode structures in the asymmetric hermean magnetosheath: Hybrid simulations, Journal of Geophysical Research: Space Physics 118 (2013) 405-417. doi:10.1029/2012JA018083.

[29] D. Herčík, P. M. Trávníček, Š. Štverák, P. Hellinger, Properties of hermean plasma belt: Numerical simulations and comparison with messenger data, Journal of Geophysical Research: Space Physics 121 (2016) 413-431. URL: https://agupubs.onlinelibrary.wiley.com/ doi/abs/10.1002/2015JA021938. doi:10.1002/2015JA021938. 
[30] S. Fatemi, A. R. Poppe, G. T. Delory, W. M. Farrell, Amitis: A 3D GPU-based hybrid-PIC model for space and plasma physics, Journal of Physics: Conference Series 837 (2017) 012017.

[31] S. Fatemi, N. Poirier, M. Holmström, J. Lindkvist, M. Wieser, S. Barabash, A modelling approach to infer the solar wind dynamic pressure from magnetic field observations inside Mercury's magnetosphere, Astronomy \& Astrophysics 614 (2018) A132. doi:10.1051/0004-6361/ 201832764.

[32] W. Exner, D. Heyner, L. Liuzzo, U. Motschmann, D. Shiota, K. Kusano, T. Shibayama, Coronal mass ejection hits Mercury: A.I.K.E.F. hybridcode results compared to messenger data, Planetary and Space Science $153(2018) 89-99$.

[33] W. Exner, S. Simon, D. Heyner, U. Motschmann, Influence of Mercury's Exosphere on the Structure of the Magnetosphere, Journal of Geophysical Research (Space Physics) 125 (2020) e27691. doi:10.1029/ 2019JA027691.

[34] J. Müller, S. Simon, Y.-C. Wang, U. Motschmann, D. Heyner, J. Schüle, W.-H. Ip, G. Kleindienst, G. J. Pringle, Origin of Mercury's double magnetopause: 3D hybrid simulation study with A.I.K.E.F., Icarus 218 (2012) 666-687. doi:10.1016/j . icarus.2011.12.028.

[35] B. J. Anderson, J. A. Slavin, H. Korth, S. A. Boardsen, T. H. Zurbuchen, J. M. Raines, G. Gloeckler, R. L. McNutt, S. C. Solomon, The dayside 
magnetospheric boundary layer at Mercury, Planetary and Space Science 59 (2011) 2037-2050. doi:10.1016/j.pss.2011.01.010.

[36] I. B. Peng, S. Markidis, E. Laure, A. Johlander, A. Vaivads, Y. Khotyaintsev, P. Henri, G. Lapenta, Kinetic structures of quasiperpendicular shocks in global particle-in-cell simulations, Physics of Plasmas 22 (2015) 092109. doi:10.1063/1.4930212.

[37] I. B. Peng, S. Markidis, A. Vaivads, J. Vencels, J. Amaya, A. Divin, E. Laure, G. Lapenta, The formation of a magnetosphere with implicit particle-in-cell simulations, Procedia Computer Science 51 (2015) 1178 1187. doi:http://dx.doi.org/10.1016/j .procs .2015 .05 .288, international Conference On Computational Science 2015: Computational Science at the Gates of Nature.

[38] Y. Chen, G. Toth, X. Jia, J. Slavin, W. Sun, S. Markidis, T. Gombosi, J. Raines, Studying dawn-dusk asymmetries of Mercury's magnetotail using mhd-epic simulations, Journal of Geophysical Research: Space Physics (Submitted) (2019).

[39] T. Mukai, H. Yamakawa, H. Hayakawa, Y. Kasaba, H. Ogawa, Present status of the bepicolombo/Mercury magnetospheric orbiter, Advances in Space Research 38 (2006) 578 - 582. doi:https ://doi .org/10.1016/ j.asr.2005.09.038, Mercury, Mars and Saturn.

[40] A. Milillo, M. Fujimoto, E. Kallio, S. Kameda, F. Leblanc, Y. Narita, G. Cremonese, H. Laakso, M. Laurenza, S. Massetti, S. McKennaLawlor, A. Mura, R. Nakamura, Y. Omura, D. A. Rothery, K. Seki, 
M. Storini, P. Wurz, W. Baumjohann, E. J. Bunce, Y. Kasaba, J. Helbert, A. Sprague, Hermean Environment WG members, The BepiColombo mission: An outstanding tool for investigating the Hermean environment, Planetary and Space Science 58 (2010) 40-60. doi:10.1016/j.pss.2008.06.005.

[41] A. Milillo, M. Fujimoto, G. Murakami, J. Benkhoff, J. Zender, S. Aizawa, M. Dósa, L. Griton, D. Heyner, G. Ho, S. M. Imber, X. Jia, T. Karlsson, R. M. Killen, M. Laurenza, S. T. Lindsay, S. McKenna-Lawlor, A. Mura, J. M. Raines, D. A. Rothery, N. André, W. Baumjohann, A. Berezhnoy, P. A. Bourdin, E. J. Bunce, F. Califano, J. Deca, S. dêA la Fuente, C. Dong, C. Grava, S. Fatemi, P. Henri, S. L. Ivanovski, B. V. Jackson, M. James, E. Kallio, Y. Kasaba, E. Kilpua, M. Kobayashi, B. Langlais, F. Leblanc, C. Lhotka, V. Mangano, A. Martindale, S. Massetti, A. Masters, M. Morooka, Y. Narita, J. S. Oliveira, D. Odstrcil, S. Orsini, M. G. Pelizzo, C. Plainaki, F. Plaschke, F. Sahraoui, K. Seki, J. A. Slavin, R. Vainio, P. Wurz, S. Barabash, C. M. Carr, D. Delcourt, K. H. Glassmeier, M. Grand e, M. Hirahara, J. Huovelin, O. Korablev, H. Kojima, H. Lichtenegger, S. Livi, A. Matsuoka, R. Moissl, M. Moncuquet, K. Muinonen, E. Quèmerais, Y. Saito, S. Yagitani, I. Yoshikawa, J. E. Wahlund, Investigating Mercury's Environment with the TwoSpacecraft BepiColombo Mission, Space Science Reviews 216 (2020) 93. doi:10.1007/s11214-020-00712-8.

[42] B. J. Anderson, C. L. Johnson, H. Korth, R. M. Winslow, J. E. Borovsky, M. E. Purucker, J. a. Slavin, S. C. Solomon, M. T. Zuber, 
R. L. McNutt, Low-degree structure in Mercury's planetary magnetic field, Journal of Geophysical Research E: Planets 117 (2012) 1-17. doi:10.1029/2012JE004159.

[43] R. Keppens, Z. Meliani, A. J. van Marle, P. Delmont, A. Vlasis, B. van der Holst, Parallel, grid-adaptive approaches for relativistic hydro and magnetohydrodynamics, Journal of Computational Physics 231 (2012) 718-744. doi:10.1016/j.jcp.2011.01.020.

[44] C. Xia, J. Teunissen, I. E. Mellah, E. Chané, R. Keppens, MPIAMRVAC 2.0 for solar and astrophysical applications, The Astrophysical Journal Supplement Series 234 (2018) 30. doi:10.3847/1538-4365/ aaa6c8.

[45] K. G. Powell, P. L. Roe, T. J. Linde, T. I. Gombosi, D. L. De Zeeuw, A Solution-Adaptive Upwind Scheme for Ideal Magnetohydrodynamics, Journal of Computational Physics 154 (1999) 284-309. doi:10.1006/ jcph.1999.6299.

[46] L. Griton, F. Pantellini, Z. Meliani, Three-dimensional magnetohydrodynamic simulations of the solar wind interaction with a hyperfastrotating uranus, Journal of Geophysical Research: Space Physics 123 (2018) 5394-5406. doi:10.1029/2018JA025331.

[47] M. Yagi, K. Seki, Y. Matsumoto, Development of a magnetohydrodynamic simulation code satisfying the solenoidal magnetic field condition, Computer Physics Communications 180 (2009) 1550 - 1557. doi:https://doi.org/10.1016/j.cpc.2009.04.010. 
[48] M. Yagi, K. Seki, Y. Matsumoto, D. C. Delcourt, F. Leblanc, Formation of a sodium ring in Mercury's magnetosphere, Journal of Geophysical Research: Space Physics 115 (2010). doi:10.1029/2009JA015226.

[49] J. Müller, S. Simon, U. Motschmann, J. Schüle, K.-H. Glaßmeier, G. J. Pringle, A.i.k.e.f.: Adaptive hybrid model for space plasma simulations, Computer Physics Communications 182 (2011) 946-966. doi:10.1016/ j.cpc.2010.12.033.

[50] J. Müller, S. Simon, Y.-C. Wang, U. Motschmann, D. Heyner, J. Schüle, W.-H. Ip, G. Kleindienst, G. J. Pringle, Origin of Mercury's double magnetopause: 3d hybrid simulation study with a.i.k.e.f., Icarus 218 (2012) 666 - 687. doi:https://doi.org/10.1016/j .icarus.2011.12. 028.

[51] C. Jacquey, V. Génot, E. Budnik, R. Hitier, M. Bouchemit, M. Gangloff, A. Fedorov, B. Cecconi, N. André, B. Lavraud, C. Harvey, F. Dériot, D. Heulet, E. Pallier, E. Penou, J. L. Pinçon, AMDA, Automated Multi-Dataset Analysis: A Web-Based Service Provided by the CDPP, Astrophysics and Space Science Proceedings 11 (2010) 239-247. doi:10.1007/978-90-481-3499-1_16.

[52] V. Génot, C. Jacquey, M. Bouchemit, M. Gangloff, A. Fedorov, B. Lavraud, N. André, L. Broussillou, C. Harvey, E. Pallier, E. Penou, E. Budnik, R. Hitier, B. Cecconi, F. Dériot, D. Heulet, J. L. Pinçon, Space Weather applications with CDPP/AMDA, Advances in Space Research 45 (2010) 1145-1155. doi:10.1016/j.asr.2009.11.010. 
[53] V. Génot, L. Beigbeder, D. Popescu, N. Dufourg, M. Gangloff, M. Bouchemit, S. Caussarieu, J. P. Toniutti, J. Durand, R. Modolo, N. André, B. Cecconi, C. Jacquey, F. Pitout, A. Rouillard, R. Pinto, S. Erard, N. Jourdane, L. Leclercq, S. Hess, M. Khodachenko, T. Al-Ubaidi, M. Scherf, E. Budnik, Science data visualization in planetary and heliospheric contexts with 3DView, Planetary and Space Science 150 (2018) 111-130. doi:10.1016/j.pss. 2017.07.007.

[54] D. A. Roberts, J. Thieman, V. Génot, T. King, M. Gangloff, C. Perry, C. Wiegand, D. De Zeeuw, S. F. Fung, B. Cecconi, S. Hess, The SPASE Data Model: A Metadata Standard for Registering, Finding, Accessing, and Using Heliophysics Data Obtained From Observations and Modeling, Space Weather 16 (2018) 1899-1911. doi:10.1029/2018SW002038.

[55] M. Sarantos, P. H. Reiff, T. W. Hill, R. M. Killen, A. L. Urquhart, A B $x^{-}$ interconnected magnetosphere model for Mercury, Planetary and Space Science 49 (2001) 1629-1635. doi:10.1016/S0032-0633(01)00100-3.

[56] E. Marsch, R. Schwenn, H. Rosenbauer, K. H. Muehlhaeuser, W. Pilipp, F. M. Neubauer, Solar wind protons: Three-dimensional velocity distributions and derived plasma parameters measured between 0.3 and 1 AU, Journal of Geophysical Research 87 (1982) 52-72. doi:10.1029/ JA087iA01p00052.

[57] M. Sarantos, J. A. Slavin, On the possible formation of alfvén wings at mercury during encounters with coronal mass ejections, Geophysical Research Letters 36 (2009). URL: https://agupubs. 
onlinelibrary .wiley . com/doi/abs/10.1029/2008GL036747. doi:10. 1029/2008GL036747.

[58] B. J. Anderson, C. L. Johnson, H. Korth, A magnetic disturbance index for Mercury's magnetic field derived from MESSENGER Magnetometer data, Geochemistry, Geophysics, Geosystems 14 (2013) 3875-3886. doi:10.1002/ggge.20242.

[59] I. Wilson, Lynn B., M. L. Stevens, J. C. Kasper, K. G. Klein, B. A. Maruca, S. D. Bale, T. A. Bowen, M. P. Pulupa, C. S. Salem, The Statistical Properties of Solar Wind Temperature Parameters Near 1 au, The Astrophysical Journal Supplement Series 236 (2018) 41. doi:10. 3847/1538-4365/aab71c. arXiv:1802.08585.

[60] J. A. Slavin, B. J. Anderson, T. H. Zurbuchen, D. N. Baker, S. M. Krimigis, M. H. Acuña, M. Benna, S. A. Boardsen, G. Gloeckler, R. E. Gold, G. C. Ho, H. Korth, R. L. McNutt Jr., J. M. Raines, M. Sarantos, D. Schriver, S. C. Solomon, P. Trávníček, Messenger observations of mercury's magnetosphere during northward imf, Geophysical Research Letters 36 (2009). URL: https://agupubs.onlinelibrary.wiley.com/ doi/abs/10 .1029/2008GL036158. doi:10.1029/2008GL036158.

[61] J. H. Shue, J. K. Chao, H. C. Fu, C. T. Russell, P. Song, K. K. Khurana, H. J. Singer, A new functional form to study the solar wind control of the magnetopause size and shape, Journal of Geophysical Research (Space Physics) 102 (1997) 9497-9512. doi:10.1029/97JA00196.

[62] G. B. Andrews, T. H. Zurbuchen, B. H. Mauk, H. Malcom, L. A. Fisk, 
G. Gloeckler, G. C. Ho, J. S. Kelley, P. L. Koehn, T. W. Lefevere, S. S. Livi, R. A. Lundgren, J. M. Raines, The Energetic Particle and Plasma Spectrometer Instrument on the MESSENGER Spacecraft, Space Science Reviews 131 (2007) 523-556. doi:10.1007/s11214-007-9272-5.

[63] W. Baumjohann, A. Matsuoka, W. Magnes, K.-H. Glassmeier, R. Nakamura, H. Biernat, M. Delva, K. Schwingenschuh, T. Zhang, H.-U. Auster, K.-H. Fornacon, I. Richter, A. Balogh, P. Cargill, C. Carr, M. Dougherty, T. S. Horbury, E. A. Lucek, F. Tohyama, T. Takahashi, M. Tanaka, T. Nagai, H. Tsunakawa, M. Matsushima, H. Kawano, A. Yoshikawa, H. Shibuya, T. Nakagawa, M. Hoshino, Y. Tanaka, R. Kataoka, B. J. Anderson, C. T. Russell, U. Motschmann, M. Shinohara, Magnetic field investigation of Mercury's magnetosphere and the inner heliosphere by MMO/MGF, Planetary and Space Science 58 (2010) 279-286. doi:10.1016/j.pss.2008.05.019.

[64] K. H. Glassmeier, H. U. Auster, D. Heyner, K. Okrafka, C. Carr, G. Berghofer, B. J. Anderson, A. Balogh, W. Baumjohann, P. Cargill, U. Christensen, M. Delva, M. Dougherty, K. H. Fornaçon, T. S. Horbury, E. A. Lucek, W. Magnes, M. Mandea, A. Matsuoka, M. Matsushima, U. Motschmann, R. Nakamura, Y. Narita, H. O’Brien, I. Richter, K. Schwingenschuh, H. Shibuya, J. A. Slavin, C. Sotin, B. Stoll, H. Tsunakawa, S. Vennerstrom, J. Vogt, T. Zhang, The fluxgate magnetometer of the BepiColombo Mercury Planetary Orbiter, Planetary and Space Science 58 (2010) 287-299. doi:10.1016/j.pss.2008.06.018.

[65] Y. Saito, J. Sauvaud, M. Hirahara, S. Barabash, D. Delcourt, 
T. Takashima, K. Asamura, Scientific objectives and instrumentation of Mercury plasma particle experiment (mppe) onboard mmo, Planetary and Space Science 58 (2010) 182 - 200. doi:https://doi.org/10.1016/ j.pss.2008.06.003, comprehensive Science Investigations of Mercury: The scientific goals of the joint ESA/JAXA mission BepiColombo.

[66] Y. Kasaba, J.-L. Bougeret, L. Blomberg, H. Kojima, S. Yagitani, M. Moncuquet, J.-G. Trotignon, G. Chanteur, A. Kumamoto, Y. Kasahara, J. Lichtenberger, Y. Omura, K. Ishisaka, H. Matsumoto, The plasma wave investigation (pwi) onboard the bepicolombo/mmo: First measurement of electric fields, electromagnetic waves, and radio waves around Mercury, Planetary and Space Science 58 (2010) 238 278. doi:https://doi.org/10.1016/j.pss.2008.07.017, comprehensive Science Investigations of Mercury: The scientific goals of the joint ESA/JAXA mission BepiColombo.

[67] S. Orsini, S. Livi, K. Torkar, S. Barabash, A. Milillo, P. Wurz, A. M. di Lellis, E. Kallio, SERENA Team, SERENA: A suite of four instruments (ELENA, STROFIO, PICAM and MIPA) on board BepiColombo-MPO for particle detection in the Hermean environment, Planetary and Space Science 58 (2010) 166-181. doi:10.1016/j.pss.2008.09.012. 

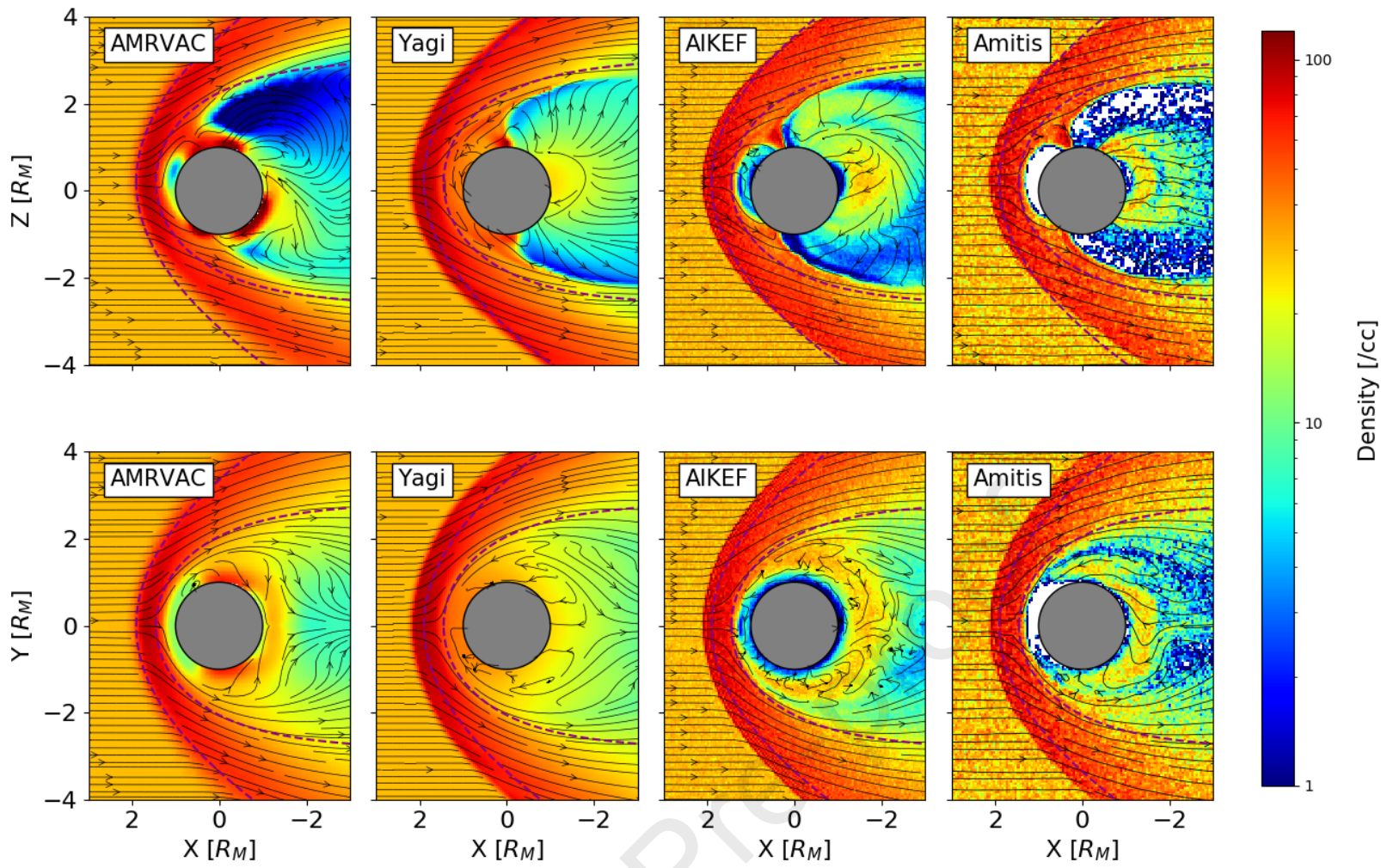


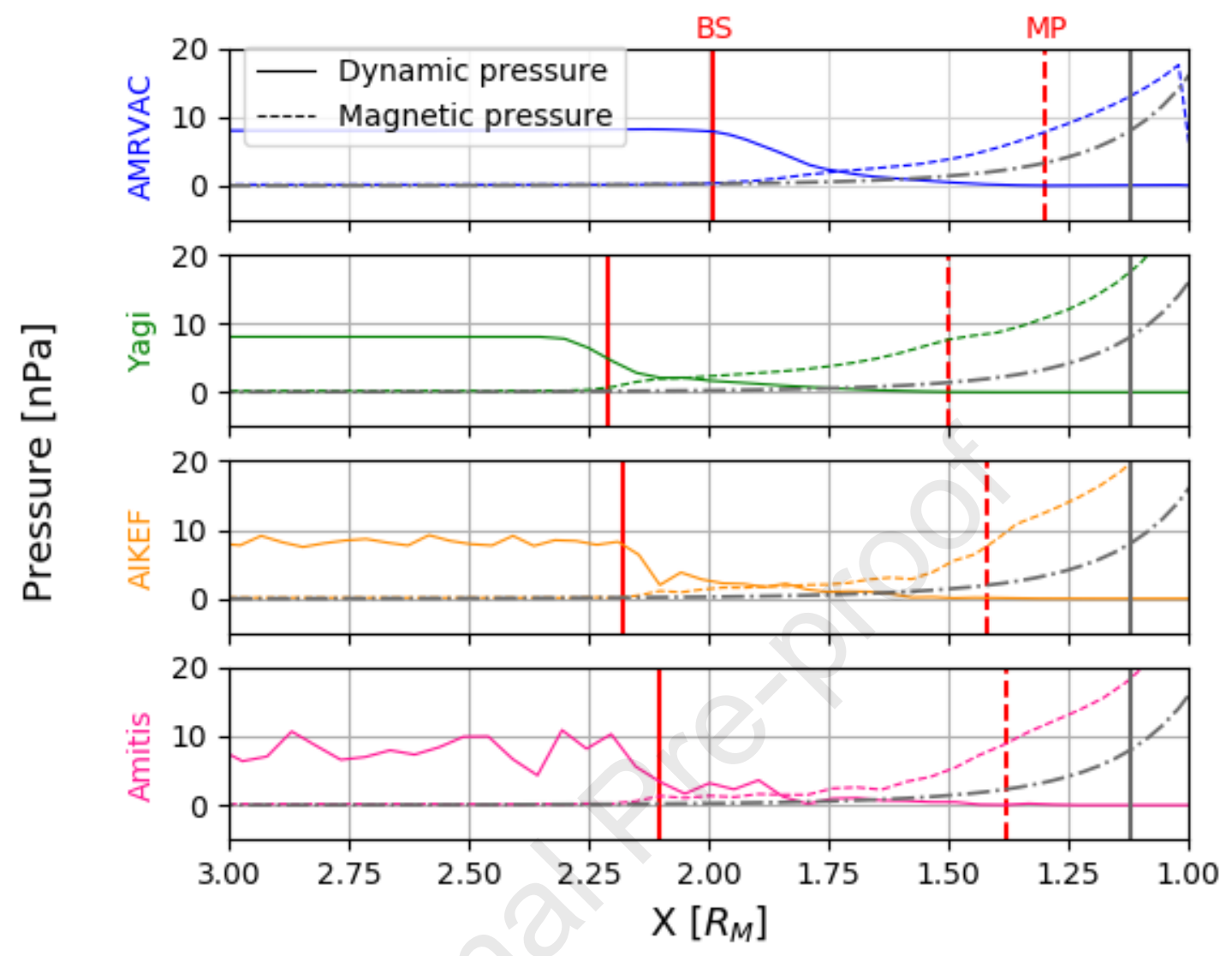




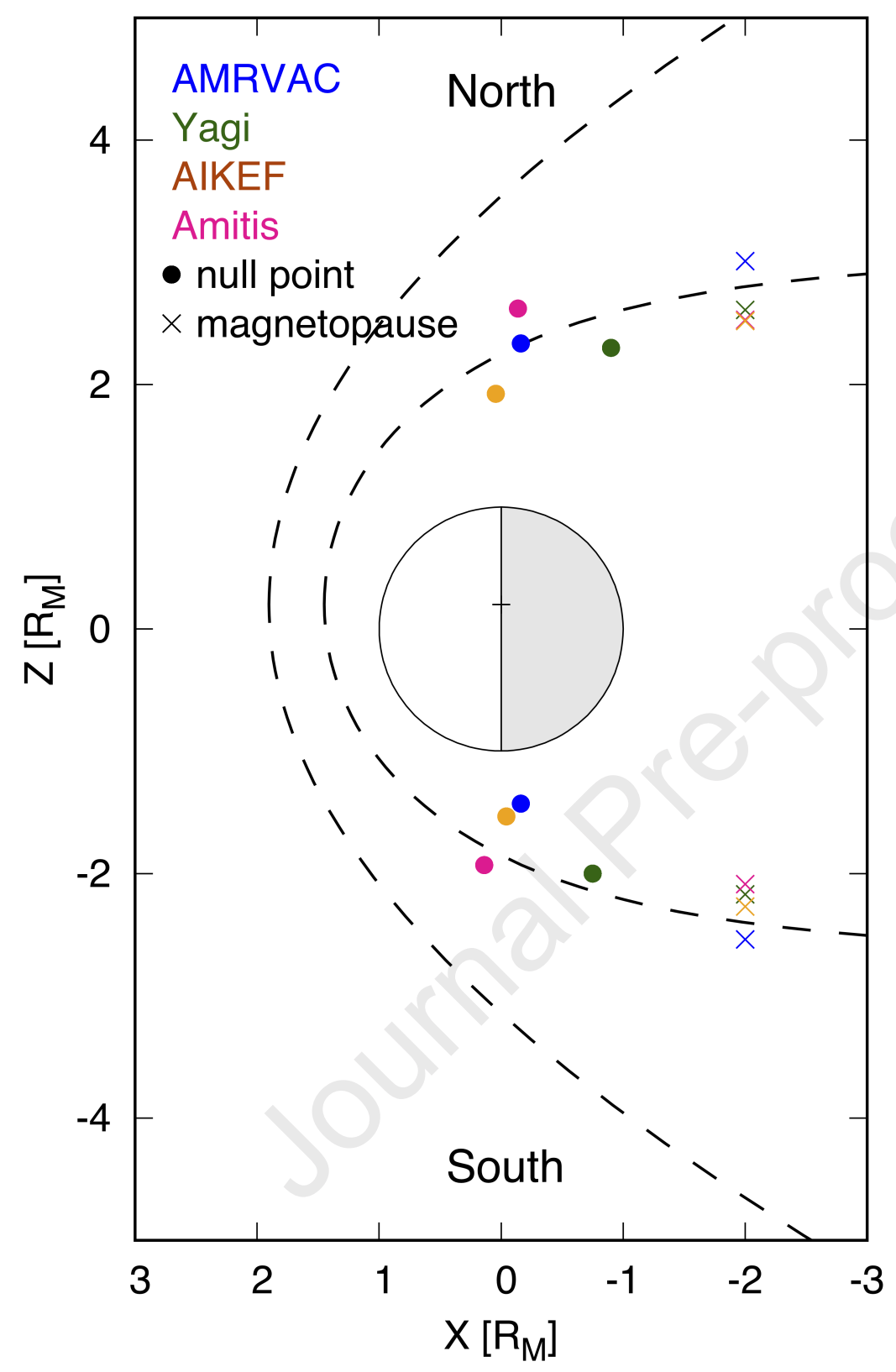



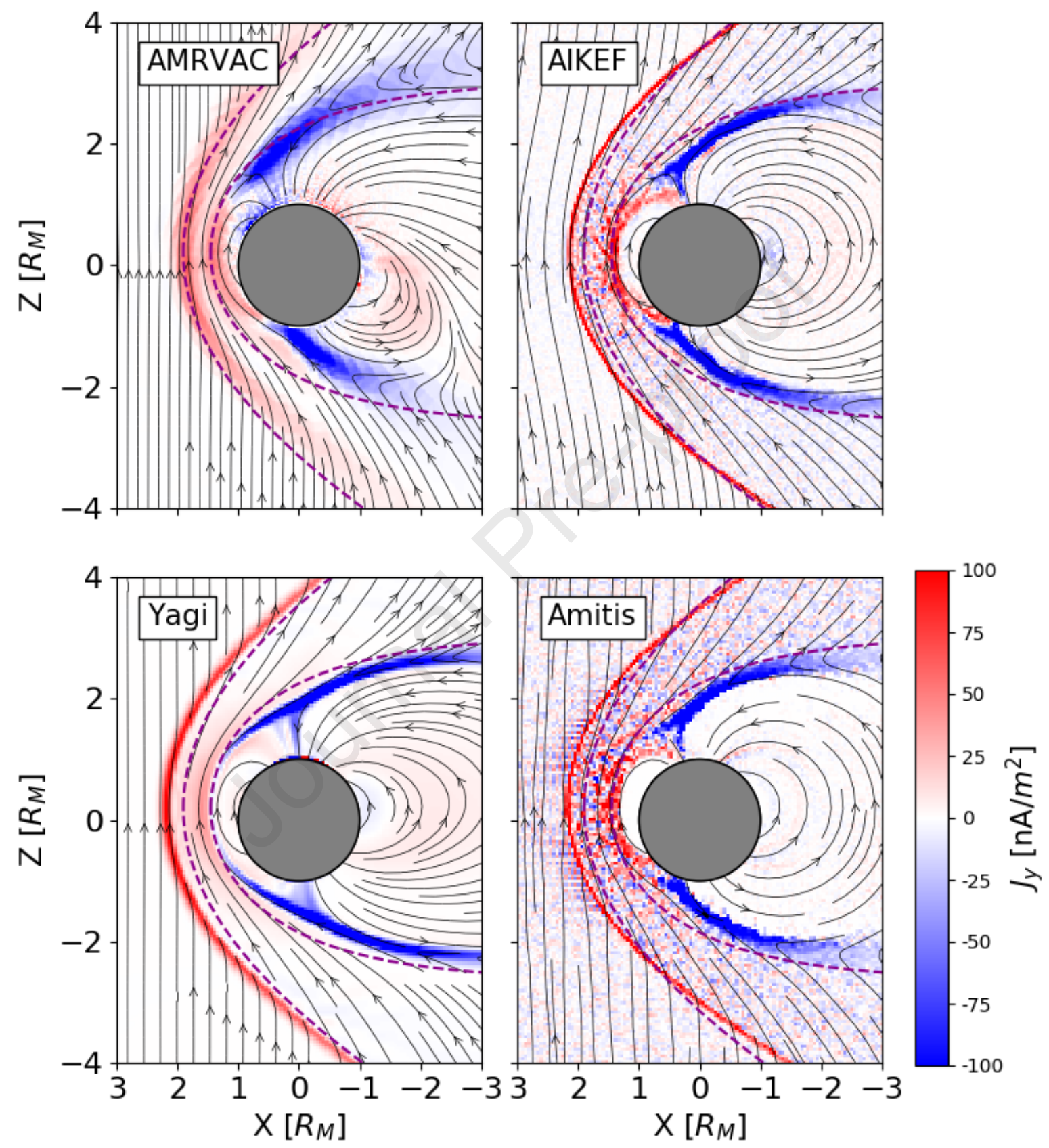

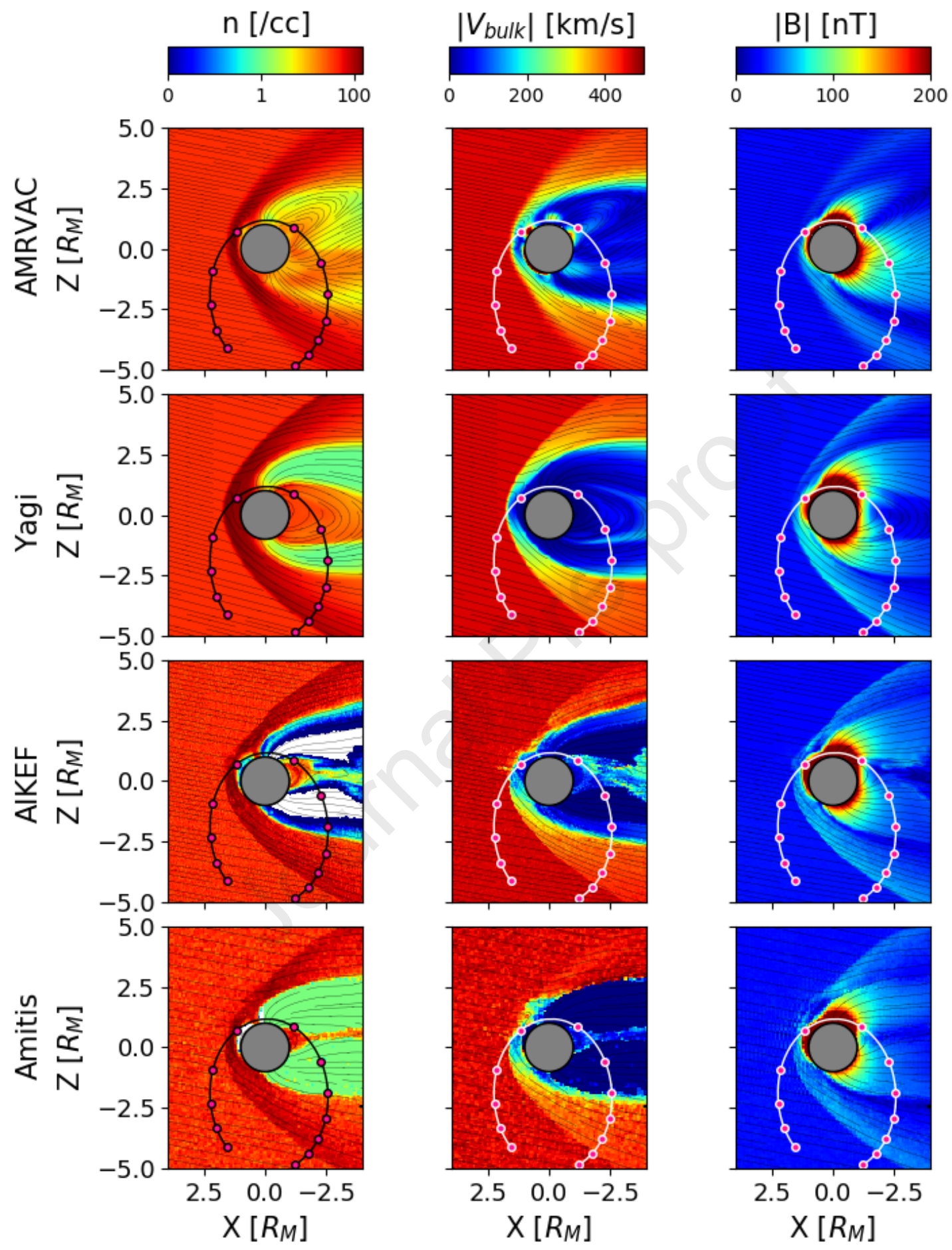







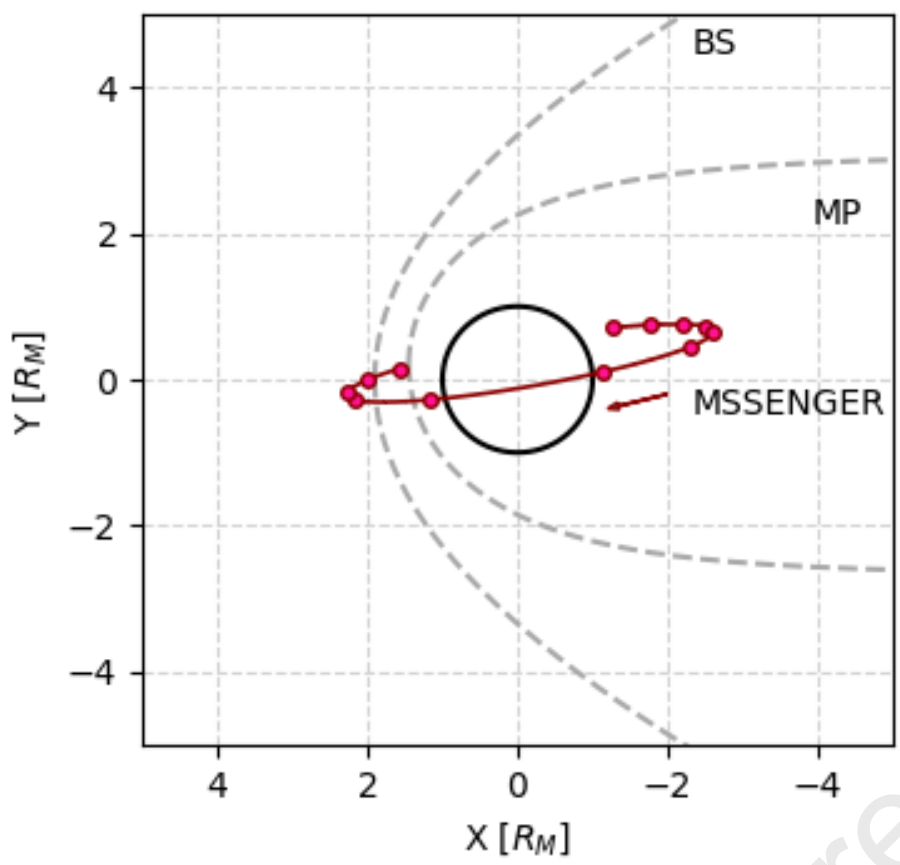




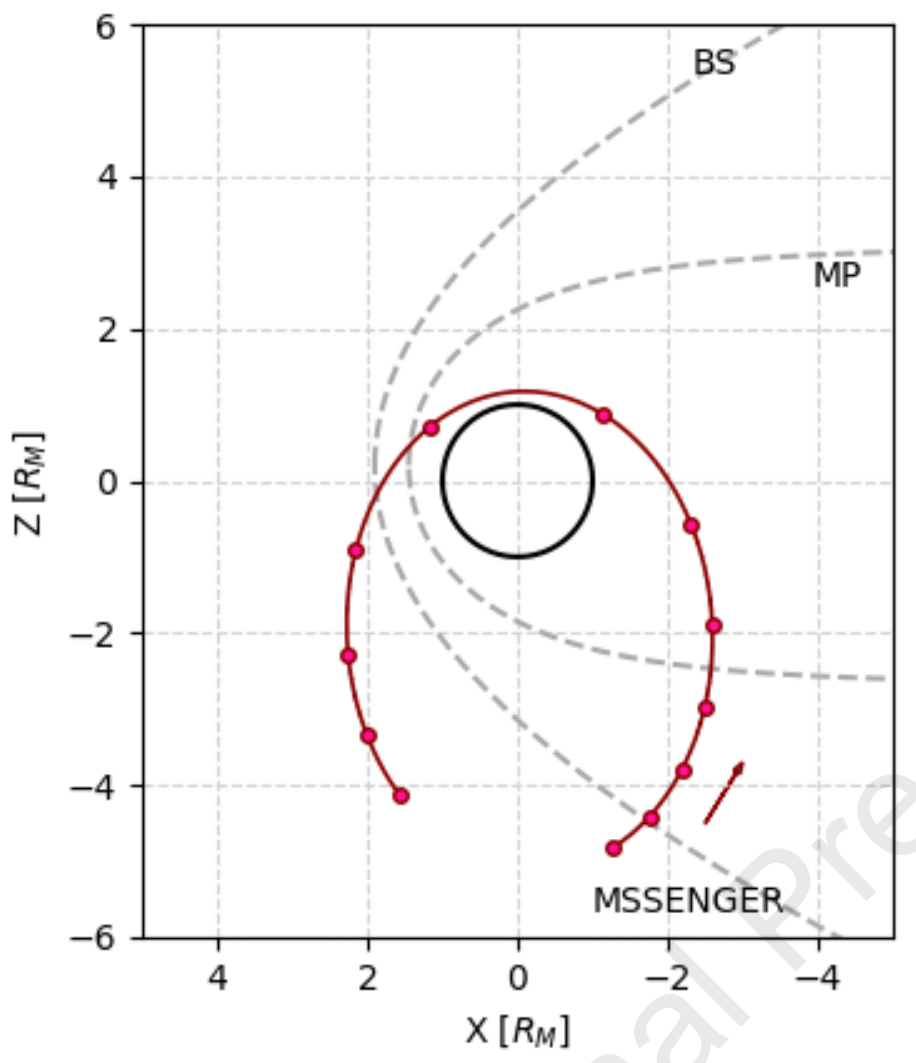


MESSENGER - 2012/11/08

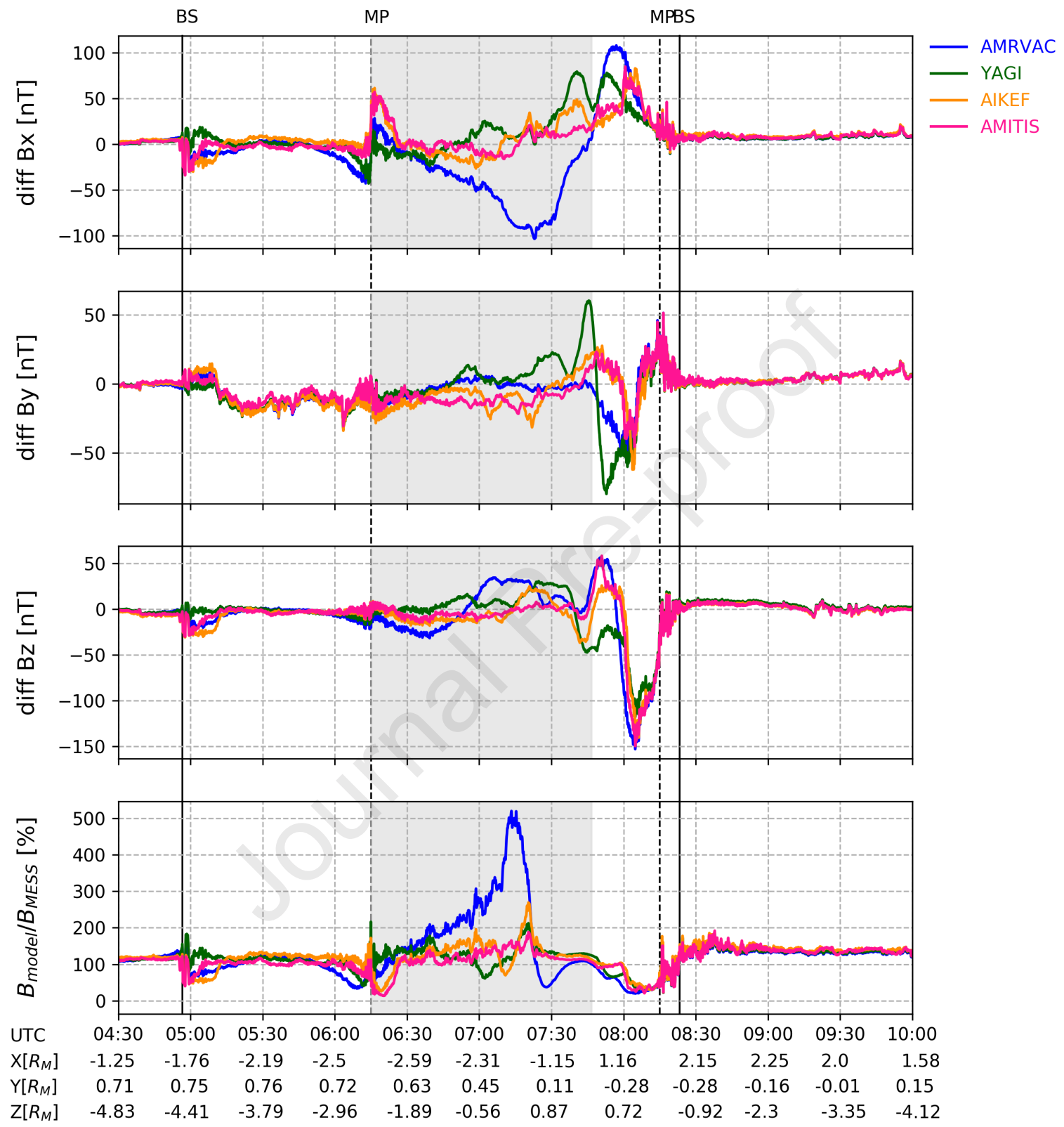




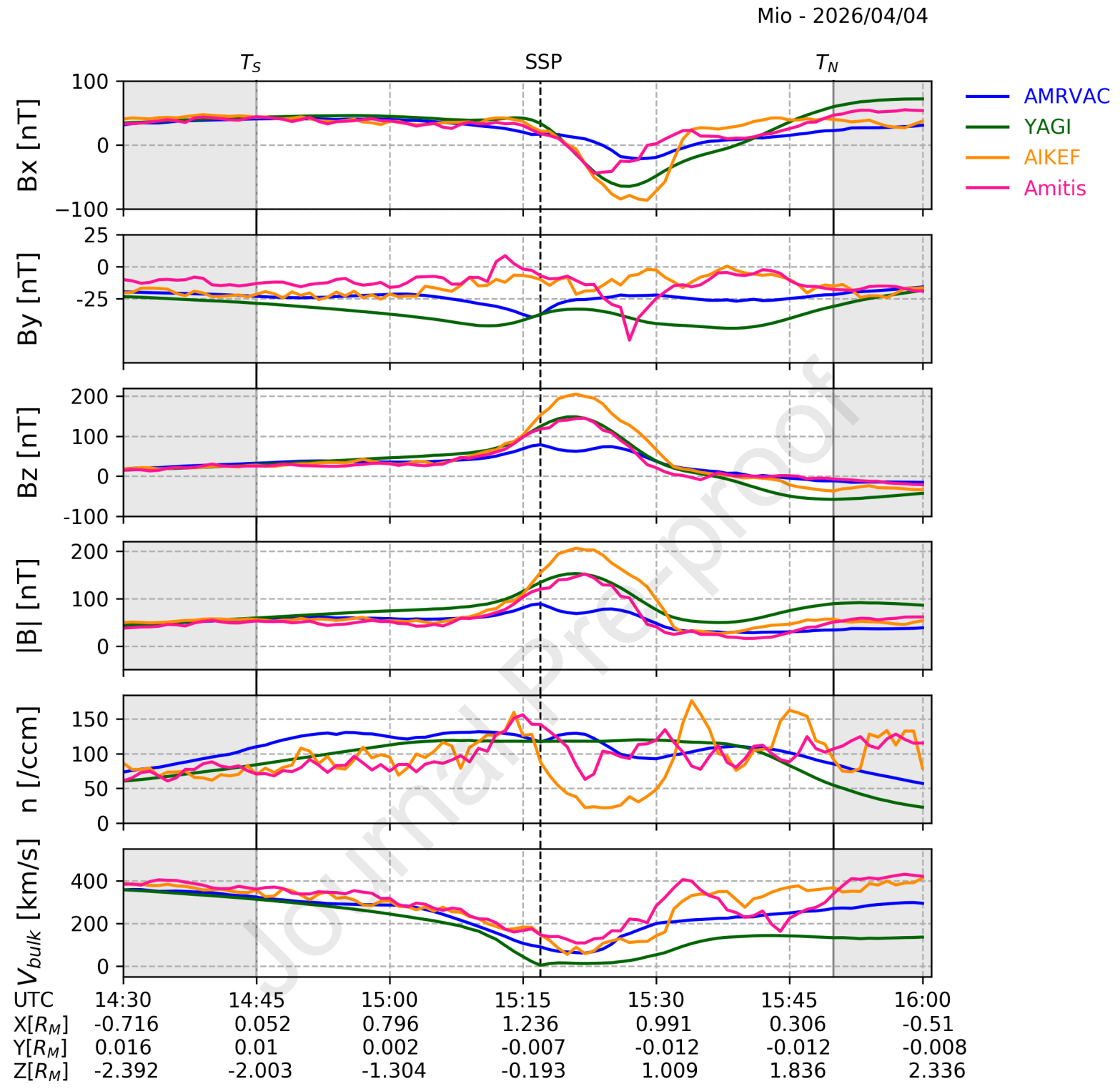




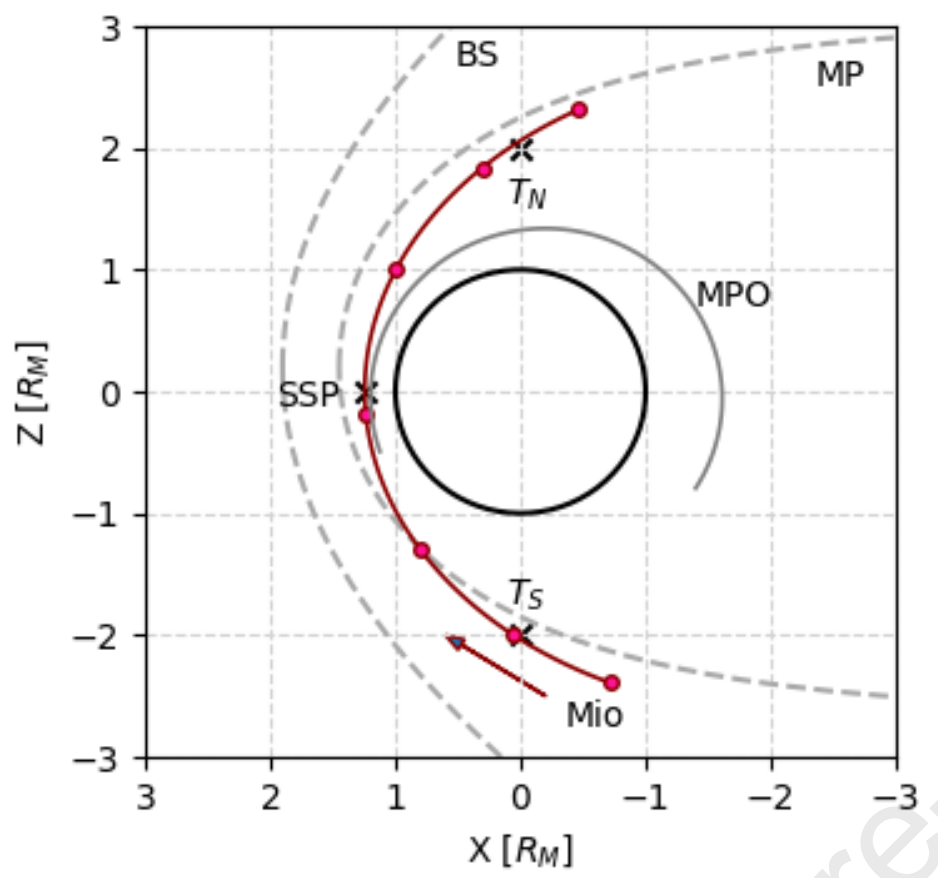




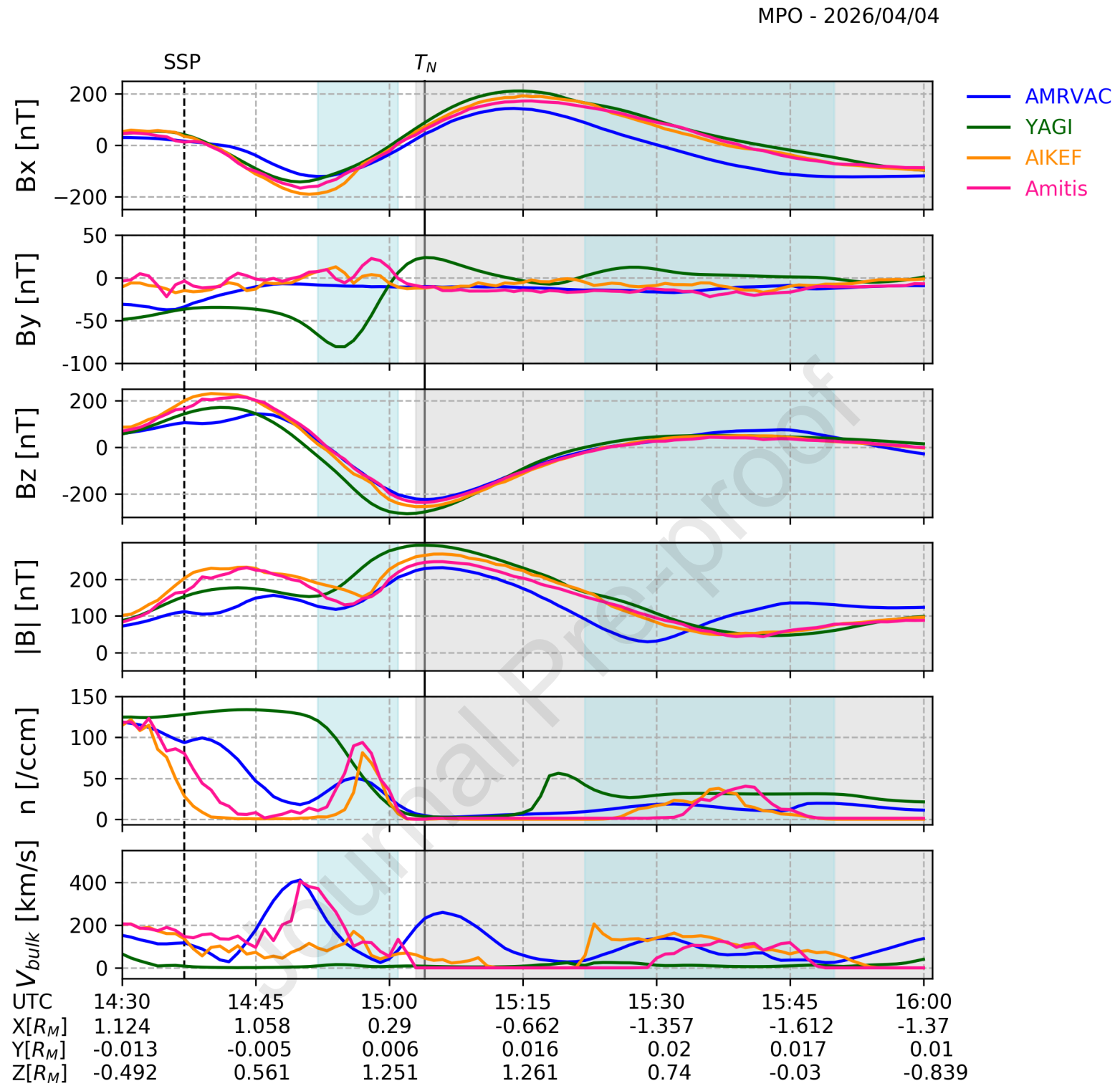




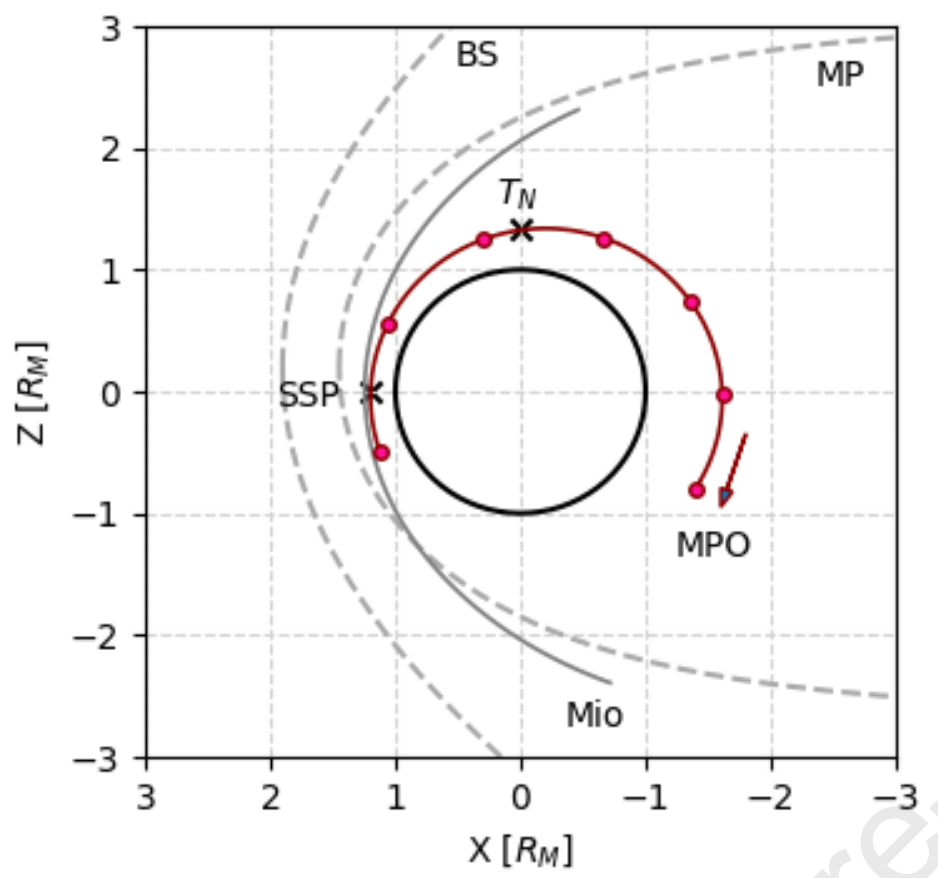




\section{Cross-comparison of global simulation models applied to} 3 Mercury's dayside magnetosphere

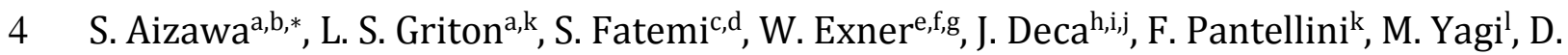
5 Heyner $^{\mathrm{e}}$, V. G'enot $^{\mathrm{a}}$, N. Andr'e ${ }^{\mathrm{a}}$, J. Amaya ${ }^{\mathrm{m}}$, G. Murakami $^{\mathrm{n}}$, L. Beigbeder ${ }^{\mathrm{O}}$, M. Gangloff ${ }^{\mathrm{a}}$, M.

6 Bouchemit $^{\mathrm{a}}$, E. Budnik ${ }^{\mathrm{p}}$, H. Usui ${ }^{\mathrm{q}}$

aIRAP, CNRS-CNES-UPS, Toulouse, France

${ }^{\mathrm{b}}$ Graduate School of Science, Tohoku University, Sendai, Japan

${ }^{\mathrm{c}}$ Swedish Institute of Space Physics, Kiruna, Sweden

${ }^{\mathrm{d}}$ Department of Physics at Ume a University, Ume a, Sweden

eInstitute for Geophysics and extraterrestrial Physics, Technische Universit”at Braunschweig, Braunschweig, Germany

f Institute for Theoretical Physics, Technische Universiẗat Braunschweig, Braunschweig, Germany

${ }^{\mathrm{g}} \mathrm{School}$ of Earth and Atmospheric Sciences, Georgia Institute of Technology, Atlanta, USA

${ }^{h}$ Laboratory for Atmospheric and Space Physics (LASP), University of Colorado Boulder, Boulder, Colorado 80303, USA

institute for Modeling Plasma, Atmospheres and Cosmic Dust, NASA/SSERVI, California 94035, USA

jLaboratoire Atmosph`eres, Milieux, Observations Spatiales (LATMOS), Universit'e de Versailles `a Saint Quentin, 78280 Guyancourt, France

${ }^{\mathrm{k}}$ LESIA, Observatoire de Paris, Universit'e PSL, CNRS, Sorbonne Universit'e, Universit'e de Paris, 5 place Jules Janssen, 92195 Meudon, France

${ }^{l}$ RIKEN, Kobe, Japan

${ }^{\mathrm{m}} \mathrm{CmPA}$, Mathematics Department, KU Leuven, Belgium

${ }^{\mathrm{n}}$ ISAS/JAXA, Sagamihara, Japan

${ }^{\circ} \mathrm{GFI}$, Toulouse, France

${ }^{\mathrm{p}}$ Noveltis, Toulouse, France

${ }^{\mathrm{q}}$ Kobe University, Kobe, Japan

Key Points :

1) First comparison of multiple global simulations of the solar wind interaction with Mercury's dayside magnetosphere

2) The results of four distinct simulation approaches are juxtaposed with MESSENGER observations

3) In contrast to the magnetosheath thickness, the simulated shock locations 


\section{Declaration of interests}

$\bigotimes$ The authors declare that they have no known competing financial interests or personal relationships that could have appeared to influence the work reported in this paper.

$\square$ The authors declare the following financial interests/personal relationships which may be considered as potential competing interests:

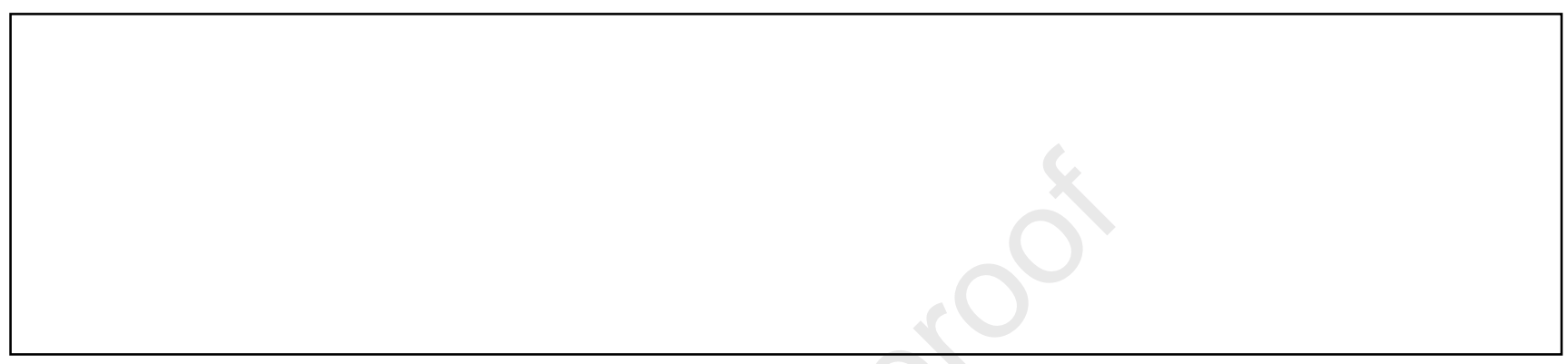

